\title{
About the Prospects for Passage to Instability
}

\author{
I. V. Lebed \\ Zhukovsky Central Institute of Aerohydrodynamics, Moscow, Russia \\ Email: lebed-i@newmail.ru
}

Received July 5, 2013; revised August 3, 2013; accepted August 17, 2013

Copyright (C) 2013 I. V. Lebed. This is an open access article distributed under the Creative Commons Attribution License, which permits unrestricted use, distribution, and reproduction in any medium, provided the original work is properly cited.

\begin{abstract}
The results of the direct numerical integration of the Navier-Stokes equations are evaluated against experimental data for problem on a flow around bluff bodies in an unstable regime. Experiment records several stable medium states for flow past a body. Evolution of each of these states, after losing the stability, inevitably goes by periodic vortex shedding modes. Calculations based on the Navier-Stokes equations satisfactorily reproduced all observed stable medium states. They were, however, incapable of reproducing any of a vortex shedding modes recorded experimentally. The solutions to the classic hydrodynamics equations successfully reach the boundary of instability field. However, classic solutions are unable to cross this boundary. Most likely, the reason for this is the Navier-Stokes equations themselves. The classic hydrodynamics equations directly follow from the Boltzmann equation and naturally contain the error involved in the derivation of classic kinetic equation. Just the Boltzmann hypothesis, which closed kinetic equation, allowed us to construct classic hydrodynamics on only three lower principal hydrodynamic values. The use of the Boltzmann hypothesis excludes higher principal hydrodynamic values from the participation in the formation of classic hydrodynamics equations. The multimoment hydrodynamics equations are constructed using seven lower principal hydrodynamic values. The numerical integration of the multimoment hydrodynamics equations in the problem on flow around a sphere shows that the solutions to these equations cross the boundary and enter the instability field. The boundary crossing is accompanied by appearance of very uncommon acts in scenario of system evolution.
\end{abstract}

Keywords: Instability; Classic Hydrodynamics; Multimoment Hydrodynamics

\section{Introduction}

The possibility to interpret unstable phenomena becomes very topical. In particular, the reason is conditioned by visualized increase in the intensity of disasters in nature. Experiment expects that the phenomenon of vortex shedding is the example of unstable process. Vortex shedding behind bluff bodies has been studied systematically at least since the days of Strouhal. Vortex shedding is welldefined instability development regime, which is fairly extended along the Reynolds scale. Sometimes, the phenomenon of vortex shedding is called the von Karman instability. Sometimes, the phenomenon is called the Kelvin-Helmholtz instability. However, the study of evolution of solution, after losing the stability, by means of direct numerical integration of the Navier-Stokes equations became feasible comparatively recently.

The phenomenon of vortex shedding behind bluff bodies is taken for evaluation of the results of direct numerical integration of the Navier-Stokes equations against experimental data [1,2]. In Section 2, the analysis of numerous divergences between the results of numerical integration of the Navier-Stokes equations and the experiment is given. The analysis is accompanied by corresponding conclusions. In Section 3, the concepts that lie in foundation of the multimoment hydrodynamics [3] are discussed. The characteristic features of unstable solution to the multimoment hydrodynamics equations in the problem on flow around a sphere $[4,5]$ are considered.

\section{Evaluating the Results of Numerical Integration against Experiment}

In each of the problems on flow around a bluff bodies experiment records several independent directions of instability development. For 3D flow past a sphere experiment records three stable medium states. The $\boldsymbol{U}_{s 0}^{\text {exp }}(\boldsymbol{x})$ stable stationary flow consists of an axisymmetric toroidal recirculating zone in the near wake, which originates a single rectilinear thread in the far wake, see Figure 1(g) in [6]. The $\boldsymbol{U}_{s 1}^{\exp }(\boldsymbol{x})$ stable nonaxisymmetric flow consists of two weakly asymmetric halves in the near wake, which originate two rectilinear threads in the far wake, see Figure 12(a) in [7]. The 
$\boldsymbol{U}_{s 2}^{e x p}(t, \boldsymbol{x})$ central-type stable state is characterized by periodic restructuring in the near non-axisymmetric wake, which causes wavy motion in the far wake without vortex shedding, see Figure 12(b) in [7].

Each of these three states, $\boldsymbol{U}_{s 0}^{\text {exp }}(\boldsymbol{x}), \boldsymbol{U}_{s 1}^{\text {exp }}(\boldsymbol{x})$, and $\boldsymbol{U}_{s 2}^{\exp }(t, \boldsymbol{x})$, after losing the stability, starts to evolve in its own direction qualitatively different from the others [2]. These directions are schematically shown by three horizontal branches in Figure 1. Instability begins to develop upon the attainment of critical Reynolds number values, $\mathrm{Re}_{0}^{*}, \mathrm{Re}_{1}^{*}$, and $\mathrm{Re}_{2}^{*}$, respectively. Instability development inevitably involves periodic vortex shedding modes. Each of the three turbulence development directions has vortex shedding features of its own only characteristic of the given direction. No matter what direction is selected by experiments, periodic vortex shedding is, however, an unavoidable, well-defined instability-development mode.

After the attainment of a certain critical Reynolds number value $\operatorname{Re}_{2}^{*}>\operatorname{Re}_{2}$, the $\boldsymbol{V}_{s 2}^{\exp }(t, \boldsymbol{x})$ one-periodic vortex loop street appears in the wake behind a sphere, the upper branch in Figure 1. The periphery of the recirculating zone is periodically detached from the core and moves downstream in the form of hairpin vortex loop. The vortex loops move uniformly along one of the paths of the double undulated thread, which forms the far wake [7]. According to experiment, two vortex shedding modes existed at $\operatorname{Re}>\operatorname{Re}_{2}^{* *}$. In the $\boldsymbol{W}_{s 2}^{\exp }(t, \boldsymbol{x})$ twoperiodic mode, hairpin vortex loops are shed, whereas the $\boldsymbol{Q}_{s 2}^{e x p}(t, \boldsymbol{x})$ two-periodic mode is characterized by vortex ring shedding. As with $\boldsymbol{W}_{s 2}^{\exp }(t, \boldsymbol{x})$, the $\boldsymbol{Q}_{s 2}^{e x p}(t, \boldsymbol{x})$ vortex rings are alternately shed from two symmetry related recirculating zone points and rush downstream along different double undulated thread paths $[7,8]$, the upper branch in Figure 1.

Above some critical $\mathrm{Re}_{1}^{*}$ value, the $\boldsymbol{U}_{s 1}^{\exp }(\boldsymbol{x})$ flow loses stability, the middle branch in Figure 1. The periphery of the recirculating zone of the $\boldsymbol{V}_{s 1}^{\exp }(t, \boldsymbol{x})$ oneperiodic flow separates from its core and moves downstream along one of the double rectilinear thread paths in the form of hairpin vortex loop [9].

After the attainment of a certain critical Reynolds number $\mathrm{Re}_{0}^{*}$ the $\boldsymbol{U}_{s 0}^{\exp }(\boldsymbol{x})$ toroidal recirculating zone in the near wake behind a sphere begins to pulsate periodically, the lower branch in Figure 1. The frequency of pulsations is anomalously low. The $\boldsymbol{V}_{s 0}^{\exp }(t, \boldsymbol{x})$ pulsating flow remains axisymmetric $[6,10]$. After the passage of $\mathrm{Re}_{0}^{* *}$, vortex rings depart from a sphere downstream and move along the spiral path, that is, the $\boldsymbol{W}_{s 0}^{\exp }(t, \boldsymbol{x})$ one-periodic flow becomes nonaxisymmetric [10]. The attainment of $\mathrm{Re}_{0}^{* * *}$ is accompanied by a change in the regime of vortex shedding from a sphere. Vortex rings penetrate into each other and form the $\boldsymbol{Q}_{s 0}^{e x p}(t, \boldsymbol{x})$ twoperiodic continuous spiral sheet in the wake behind a sphere [10], the lower branch in Figure 1.

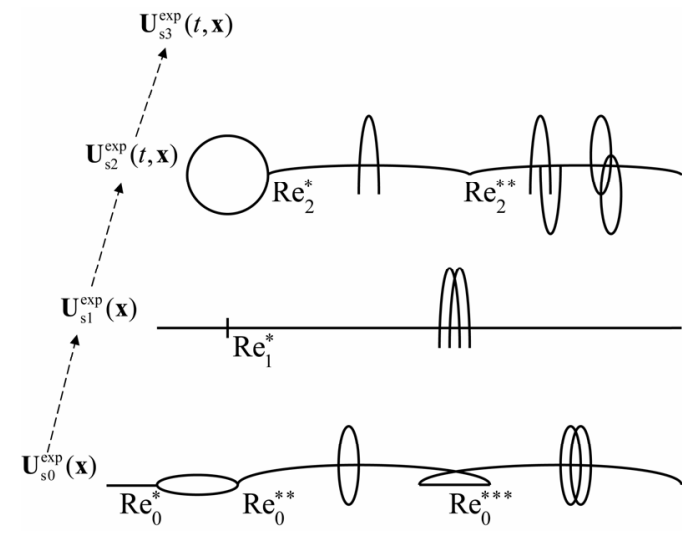

Figure 1. Three stable medium states originating three turbulence development directions for flow past a sphere. The lower branch corresponds to the evolution of stationary axisymmetric flow $U_{s 0}^{\exp }(x): \operatorname{Re}<\operatorname{Re}_{0}^{*}-U_{s 0}^{\exp }(x)$;

$\operatorname{Re}_{0}^{*}<\operatorname{Re}<\operatorname{Re}_{0}^{* *}$, periodic pulsations of the axisymmetric recirculating zone in the wake behind a sphere $V_{s 0}^{e x p}(t, x)$, see Figure 1(h) in [6]; $\operatorname{Re}_{0}^{* *}<\operatorname{Re}<\operatorname{Re}_{0}^{* * * *}$, vortex ring shedding along a spiral path $W_{s 0}^{e x p}(t, x)$, see Figure 2(a) in [10]; and $\operatorname{Re}>\operatorname{Re}_{0}^{* * * *}$, helicoidal vortex sheet $Q_{s 0}^{\text {exp }}(t, x)$, see Figure 2(b) in [10]. The middle branch corresponds to the evolution of stable steady nonaxisymmetric flow $U_{s 1}^{e x p}(x)$ :

$\operatorname{Re}_{1}<\operatorname{Re}<\operatorname{Re}_{1}^{*}-U_{s 1}^{e x p}(x) ; \quad \operatorname{Re}>\operatorname{Re}_{1}^{*}$, periodic horseshoe-shaped vortex loop shedding along a rectilinear path $V_{s 1}^{e x p}(t, x)$, see Figure 6 in [9]. The upper branch corresponds to the evolution of a stable central-type state $U_{s 2}^{e x p}(x): \operatorname{Re}_{2}<\operatorname{Re}_{2}<\operatorname{Re}_{2}^{*}-U_{s 2}^{e x p}(t, x) ; \quad \operatorname{Re}_{2}^{*}<\operatorname{Re}<\operatorname{Re}_{2}^{* *}$, periodic horseshoe-shaped vortex loop shedding along one of the double undulated thread branches $V_{s 2}^{e x p}(t, x)$, see

Figure 7(a,i) in [7]; and $\operatorname{Re}>\operatorname{Re}_{2}^{* *}$, periodic vortex loop shedding along both double undulated thread branches $W_{s 2}^{e x p}(t, x)$, see Figure 4 in [8] and Figure 7(a,iii) in [7], or periodic vortex ring shedding $Q_{s 2}^{\exp }(t, x)$, see Figure 6 in $[8]$.

For 2D flow past a circular cylinder experiment records two stable medium states. The $\boldsymbol{U}_{c 0}^{\exp }(\boldsymbol{x})$ stable stationary flow is characterized by axisymmetric recirculating zone in the near wake. The $\boldsymbol{U}_{c 2}^{\exp }(t, \boldsymbol{x})$ centraltype stable state is characterized by periodic restructuring in the near nonaxisymmetric wake without vortex shedding. Experiment finds at least two independent directions of instability development. Vortex shedding along each of the two directions, $\boldsymbol{V}_{c 0}^{\exp }(\boldsymbol{x})$ and $\boldsymbol{V}_{c 2}^{\exp }(t, \boldsymbol{x})$ $[11,12]$, is characterized by its own characteristic features intrinsic in it.

Evaluating the results of the direct numerical integration of the Navier-Stokes equations against experiment for unstable flows became feasible only recent twenty- 
five years, thanks to the vigorous development of computer facilities. As distinct from the earlier studies, the direct numerical integrations of the Navier-Stokes equations performed recently directly reproduce unstable flow conditions without artificially introducing non-stationary features into the stationary problem. Under artificial modeling a problem with time independent boundary conditions after stability loss is substituted by non-stationary stable problem $[13,14]$. Such substitution is need of initial conditions for non-stationary problem. The initial conditions consist of a solution for a fully developed vortex-shedding flow field. It is necessary to attract experimental data to construct such conditions. In particular, experiment must give eddy sizes and eddy positions in space, initial eddy velocities and vortex shedding frequency, beginning and completion of vortex shedding process at the Reynolds scale. To summarize, such modeling draws a picture of eddies movement. It is incapable of showing the direction of instability development.

Under the linear analysis of stability, the NavierStokes equations are linearized for small hydrodynamic values perturbations. Perturbations are expanded in Fourier series in time $[15,16]$. The linear character of studies does not allow evolution calculations to be extended to long times. That is, the linear analysis is incapable of defining the final stable saturated state. However, the linear analysis predicts characteristics of final solution, in particular, the oscillation frequencies around a stable position.

The direct numerical integration of the Navier-Stokes equations gives three stable solutions for flow around a sphere, $\boldsymbol{U}_{s 0}^{\text {cal }}(\boldsymbol{x}), \boldsymbol{U}_{s 1}^{\text {cal }}(\boldsymbol{x})$, and $\boldsymbol{U}_{s 2}^{\text {cal }}(t, \boldsymbol{x})$. These solutions reproduced three stable flows observed experimentally, $\boldsymbol{U}_{s 0}^{\exp }(\boldsymbol{x}), \boldsymbol{U}_{s 1}^{\exp }(\boldsymbol{x})$, and $\boldsymbol{U}_{s 2}^{\exp }(t, \boldsymbol{x})$, correspondently. In all calculations without exception performed by the direct numerical integration of the NavierStokes equations, instability development occurs in strict correspondence to classic Landau-Hopf scenario [17]. The calculation leads the instability development process in the direction given by dashed slanting line in Figure 1. The $\boldsymbol{U}_{s 0}^{\text {cal }}(\boldsymbol{x})$ solution, when loses stability, experiences bifurcation to the $\boldsymbol{U}_{s 1}^{\text {cal }}(\boldsymbol{x})$ solution (regular bifurcation), and the $\boldsymbol{U}_{s 1}^{\text {cal }}(\boldsymbol{x})$ solution after stability loss experiences bifurcation to the $\boldsymbol{U}_{s 2}^{c a l}(t, \boldsymbol{x})$ limiting cycle (Hopf bifurcation). The $\boldsymbol{U}_{s 2}^{\text {cal }}(t, \boldsymbol{x})$ solution loses stability to be replaced by the $\boldsymbol{U}_{s 3}^{\text {cal }}(t, \boldsymbol{x})$ chaotic solution [18-21].

The direct numerical integration of the Navier-Stokes equations gives two stable solutions for flow around a cylinder, $\boldsymbol{U}_{c 0}^{c a l}(\boldsymbol{x})$ and $\boldsymbol{U}_{c 2}^{c a l}(t, \boldsymbol{x})$. These solutions reproduced two stable flows observed experimentally, $\boldsymbol{U}_{c 0}^{\exp }(\boldsymbol{x})$ and $\boldsymbol{U}_{c 2}^{\exp }(t, \boldsymbol{x})$, correspondently. In accordance with Landau-Hopf scenario, the $\boldsymbol{U}_{c 0}^{\text {cal }}(\boldsymbol{x})$ solution, when loses stability, experiences bifurcation to the $\boldsymbol{U}_{c 2}^{c a l}(t, \boldsymbol{x})$ limiting cycle. The $\boldsymbol{U}_{c 2}^{c a l}(t, \boldsymbol{x})$ solution loses stability to be replaced by the $\boldsymbol{U}_{c 3}^{c a l}(t, \boldsymbol{x})$ chaotic solution [22].

The bifurcation of a stationary state after stability loss to a limiting cycle is represented in Figure 2. The Figure 2 was drawn for a flat plate [23]. Later, qualitatively analogous pictures were drawn for different bluff bodies [24]. The Figure 2 gives the temporal development of the A absolute value of the velocity disturbance amplitude at an isolated point in the wake of a bluff body. After the attainment of the time moment $t=0$ lower solution loses its stability. They are the $\boldsymbol{U}_{c 0}^{\text {cal }}(\boldsymbol{x})$ solution for a cylinder and the $\boldsymbol{U}_{s 1}^{\text {cal }}(\boldsymbol{x})$ solution for a sphere. By the time moment $t=t_{2}$ increasing of disturbances results in saturation of a new stable solution, namely, the non-stationary periodic limiting cycle. They are the $\boldsymbol{U}_{c 2}^{c a l}(t, \boldsymbol{x})$ solution for a cylinder and the $\boldsymbol{U}_{s 2}^{\text {cal }}(t, \boldsymbol{x})$ solution for a sphere. In accordance with definition [23], unstable regime is placed within the time interval between $t=0$ and $t=t_{2}$. So, unstable regime is limited at time. On the contrary, the visualized process of vortex shedding is not limited at time. That is, experiment records a vortex street as long as investigator wants. Moreover, unstable regime, Figure 2, is not a periodic one, because the A disturbance amplitude grows. As a result, time limited non-periodic unstable regime can not be put in correspondence to strictly periodic phenomenon of vortex shedding which has no time frameworks. So, classic hydrodynamics is incapable to come up the expectations of experiment on unstable nature of vortex shedding phenomenon.

The classic calculation can put only stable solution in correspondence to vortex shedding phenomenon. As noted above, the classic hydrodynamics equations in problem on flow past a sphere have four solutions, $\boldsymbol{U}_{s 0}^{\text {cal }}(\boldsymbol{x}), \boldsymbol{U}_{s 1}^{\text {cal }}(\boldsymbol{x}), \boldsymbol{U}_{s 2}^{\text {cal }}(t, \boldsymbol{x})$ and $\boldsymbol{U}_{s 3}^{\text {cal }}(t, \boldsymbol{x})$. And these four solutions must describe ten observed modes, $\boldsymbol{U}_{s 0}^{e x p}(\boldsymbol{x}), \boldsymbol{V}_{s 0}^{\exp }(t, \boldsymbol{x}), \boldsymbol{W}_{s 0}^{\exp }(t, \boldsymbol{x})$ and $\boldsymbol{Q}_{s 0}^{\exp }(t, \boldsymbol{x}) ;$

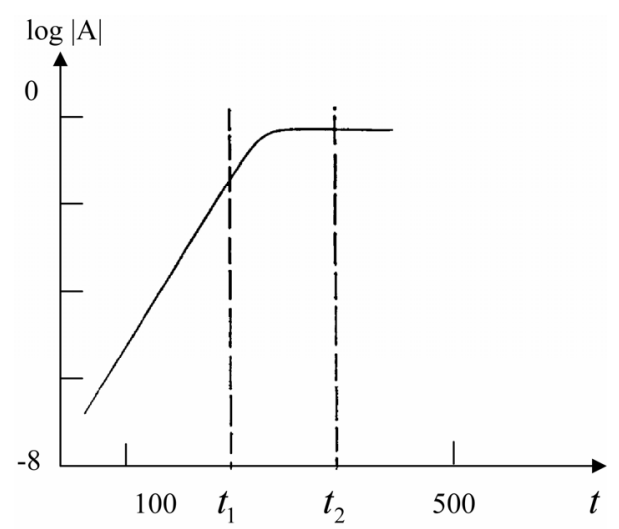

Figure 2. The temporal development of the absolute value of the velocity disturbance amplitude A: $0<t<t_{1}$ - exponential growth; $t_{1}<t<t_{2^{-}}$onset of nonlinearity; $t>t_{2^{-}}$- saturation state. Figure 2 was drawn using the data from [23]. 
$\boldsymbol{U}_{s 1}^{e x p}(\boldsymbol{x})$ and $\boldsymbol{V}_{s 1}^{\exp }(t, \boldsymbol{x}) ; \boldsymbol{U}_{s 2}^{\exp }(t, \boldsymbol{x}), \boldsymbol{V}_{s 2}^{\exp }(t, \boldsymbol{x})$, $\boldsymbol{W}_{s 2}^{\exp }(t, \boldsymbol{x})$ and $\boldsymbol{Q}_{s 2}^{\text {exp }}(t, \boldsymbol{x})$. The $\boldsymbol{U}_{s 3}^{\text {cal }}(t, \boldsymbol{x})$ solution that succeeds the $\boldsymbol{U}_{s 2}^{\text {cal }}(t, \boldsymbol{x})$ one-period mode is multiperiodic, that is, chaotic in essence. Attempts at correlating the $\boldsymbol{U}_{s 3}^{\text {cal }}(t, \boldsymbol{x})$ mode with the observed strictly periodic vortex shedding modes seem to have no prospects. The $\boldsymbol{U}_{s 0}^{\text {cal }}(\boldsymbol{x})$ solution and the $\boldsymbol{U}_{s 1}^{\text {cal }}(\boldsymbol{x})$ solution are stationary. That is why, the $\boldsymbol{U}_{s 2}^{\text {cal }}(t, \boldsymbol{x})$ limiting cycle is the only case where it is possible to correlate the observed vortex shedding from a sphere to calculation results.

Let two experimental modes have pretensions to be described by the $\boldsymbol{U}_{s 2}^{\text {cal }}(t, \boldsymbol{x})$ limiting cycle. They are the $\boldsymbol{U}_{s 2}^{e x p}(t, \boldsymbol{x})$ central-type stable state, which characterizes itself by wavy motion in the far wake without vortex shedding, and the $\boldsymbol{V}_{s 2}^{\text {exp }}(t, \boldsymbol{x})$ one-period vortex shedding mode, which manifests itself as a vortex loop street. Then, the $\boldsymbol{U}_{s 2}^{\exp }(t, \boldsymbol{x})$ central-type state does not stand out as a separate regime when the experimental data are interpreted in [18-21]. The $\boldsymbol{U}_{s 2}^{\exp }(t, \boldsymbol{x})$ central-type regime is combined with the $\boldsymbol{V}_{s 2}^{\exp }(t, \boldsymbol{x})$ regime of periodic vortex shedding from a sphere. According to [18-21], vortex shedding does occur over the Reynolds number range $\operatorname{Re}_{2}<\operatorname{Re}<\operatorname{Re}_{2}^{*}$, but its manifestations are exceedingly indistinct; the intensity of vortex shedding monotonically increases as Re grows. Earlier, this point of view was formulated in [25]. An attempt was made [18-21] to put the flow picture representing the $\boldsymbol{U}_{s 2}^{c a l}(t, \boldsymbol{x})$ limiting process in correspondence with the observed $\boldsymbol{V}_{s 2}^{\text {exp }}(t, \boldsymbol{x})$ periodic vortex shedding combined with the $\boldsymbol{U}_{s 2}^{s 2 x}(t, \boldsymbol{x})$ central-type mode.

Attempts at correlating the $\boldsymbol{U}_{s 2}^{\text {cal }}(t, \boldsymbol{x})$ solution with observed vortex shedding modes initially seem to have no prospects. The reasons are as follows. First, the suggestion of a monotonic increase in the intensity of vortex shedding [18-21] is at variance with experiment (see Figures 12(b)-12(d) in [7]). According to Figure 12(c) from [7], the vortex structure appears in the wake suddenly and is fairly well defined starting with the moment of its origination. Secondly, in accordance with calculations, the $\boldsymbol{U}_{s 3}^{\text {cal }}(t, \boldsymbol{x})$ chaotic solution replaces the $\boldsymbol{U}_{s 2}^{\text {cal }}(t, \boldsymbol{x})$ solution after the attainment of the Reynolds number value approximately equals to 500 [21]. However, in accordance with experiment, periodic vortex shedding is a well-defined mode, which is fairly extended along the Reynolds scale. In the experiments when a sphere was towed through an unperturbed medium vortex shedding was observed over the whole range of Reynolds numbers studied, up to $\operatorname{Re}=30000$ [10]. i.e., calculation predicts the early transition to chaos. This prediction is, however, at variance with experiment. Thirdly, three regimes of the six vortex shedding regimes actually observed are the one-period modes. These include $\boldsymbol{W}_{s 0}^{\text {exp }}(t, \boldsymbol{x})$, $\boldsymbol{V}_{s 1}^{\exp }(t, \boldsymbol{x})$, and $\boldsymbol{V}_{s 2}^{\exp }(t, \boldsymbol{x})$ regimes. Three regimes of vortex shedding are the two-period modes. These include $\boldsymbol{Q}_{s 0}^{\exp }(t, \boldsymbol{x}), \boldsymbol{W}_{s 2}^{\exp }(t, \boldsymbol{x})$, and $\boldsymbol{Q}_{s 2}^{\exp }(t, \boldsymbol{x})$ regimes. Attempts at correlating the one-period $\boldsymbol{U}_{s 2}^{\text {cal }}(t, \boldsymbol{x})$ solution with the observed two-period vortex shedding regimes seem to have no prospects. And at last, the $\boldsymbol{U}_{s 2}^{\text {cal }}(t, \boldsymbol{x})$ solution must be put in correspondence to several modes of vortex shedding simultaneously. For example, at some $\mathrm{Re}>\mathrm{Re}_{0}^{* * *}$, experiment records four different regimes of vortex shedding: the $\boldsymbol{Q}_{s 0}^{\text {exp }}(t, \boldsymbol{x})$ mode at the lower branch, the $\boldsymbol{V}_{s 1}^{\text {exp }}(t, \boldsymbol{x})$ mode at the middle branch, the $\boldsymbol{W}_{s 2}^{e x p}(t, \boldsymbol{x})$ and the $\boldsymbol{Q}_{s 2}^{\text {exp }}(t, \boldsymbol{x})$ modes at the upper branch, Figure 1. However, the only $\boldsymbol{U}_{s 2}^{c a l}(t, \boldsymbol{x})$ solution is incapable of reproducing four different modes simultaneously.

The streamline flow pictures for every quarter of the period of oscillations (Figure 25 in [20]) give an idea of the total period of recirculating zone oscillations in the $\boldsymbol{U}_{s 2}^{c a l}(t, \boldsymbol{x})$ wake behind a sphere after the passage of the $\mathrm{Re}_{2}^{*}$ critical value. A detailed examination of these flow pictures was executed in $[2,20]$. To summarize, after the appearance in the wake behind a sphere, the size of the vortex structure becomes substantial at the surface of the sphere. Subsequently, the structure moves toward the periphery of the recirculating zone, which is accompanied by its continuous dissipation. Lastly, it fully disappears at the periphery of the recirculating zone.

This picture is qualitatively different from the observed full period of oscillation of the recirculating zone. As in calculations, the experimental vortex structure engendered begins to expand and move downstream. After reaching the periphery of the recirculating zone, this vortex structure, however, acquires a maximum size rather than dissipates as predicted by calculations. At the end of the period, the vortex localized at the periphery of the recirculating zone separates from this zone. The separation of the shed vortex from the recirculating zone is very clearly shown in Figure 40 in [20]. The periphery of the recirculating zone, which is periodically shed from the recirculating zone, rushes downstream and forms a vortex street $\boldsymbol{V}_{s 2}^{\exp }(t, \boldsymbol{x}) \quad$ (Figure 7(a,i) in [7]).

Because of the absence of the detachment of the recirculating zone periphery in Figure 25 in the calculation [20], there is no vortex loop street in the wake behind a sphere. This is an essential difference between calculation and experiment. Analyzed Figure 25 from [20], the authors confirmed the disappearance of the vortex structure at the periphery of the recirculating zone. They note that the vortex structure "loses its spiral shape" at the periphery of the recirculating zone.

So, combining of the $\boldsymbol{U}_{s 2}^{\exp }(t, \boldsymbol{x})$ and the $\boldsymbol{V}_{s 2}^{\exp }(t, \boldsymbol{x})$ did not give the desired result, because it did not allow the calculated $\boldsymbol{U}_{s 2}^{\text {cal }}(t, \boldsymbol{x})$ mode to be put in correspondence to the observed $\boldsymbol{V}_{s 2}^{\text {exp }}(t, \boldsymbol{x})$ vortex shedding mode. Indeed, the calculated $\boldsymbol{U}_{s 2}^{c a l}(t, \boldsymbol{x})$ limiting cycle satis- 
factorily reproduces the stable central-type position $\boldsymbol{U}_{s 2}^{\exp }(t, \boldsymbol{x})$ at $\mathrm{Re}<\mathrm{Re}_{2}^{*}$ but proves a complete failure when attempts are made to reproduce the $\boldsymbol{V}_{s 2}^{\text {exp }}(t, \boldsymbol{x})$ vortex shedding mode at $\operatorname{Re}>\operatorname{Re}_{2}^{*}$ [18-21].

The $\boldsymbol{U}_{s 2}^{\text {cal }}(t, \boldsymbol{x})$ wake behind a sphere is drawn in [20] both by streamline flow pictures and by streakline flow pictures. On the flow pictures presented by streaklines, the lines having a form of hairpin vortices appear in near wake behind a sphere. However, a vortex street is absent too in the far wake on flow pictures presented by streaklines.

The authors of all the numerical experiments without exception discover the evident absence of a vortex street in the wake behind different bluff bodies on flow pictures. Numerical simulations were performed both for 2D and for 3D problems on flow around a circular cylinder and a flat plate, around a sphere and a disk, and so on. However, passed years gave no numerical experiments in which stability loss results in a periodic vortex shedding mode. Then, the conclusion of agreement between calculation and experiment is based in early direct numerical simulations on a comparison of the calculated vorticity distributions with visual observation results [18,21]. However, even insignificant twist in a streamline can create closed curves in the vorticity distribution pictures. And, really, the comparison of streamline flow pictures with the vorticity distributions (Figures 25 and 29 in [20]) shows that closed curves in the vorticity pictures correspond to wavy motion in the far wake behind a sphere rather than vortex structures. So, there is no reason at all for identifying the closed vorticity distribution curves in the wake with vortex structures.

That is why, Johnson and Patel [20] do not confine to drawing of streamline curves, streakline curves and vorticity distribution curves. To interpret the obvious contradiction between the observed vortex shedding and calculation results they put the regions of the supposed existence of vortex structures $\lambda_{2}(t, \boldsymbol{x})$ in correspondence to the observed vortex shedding. The latter direct numerical simulations select namely this way to interpret the discrepancies between calculation and experiment $[26,27]$. The sense is that both calculated and experimental flow pictures are known to change qualitatively depending on the system of coordinates used to observe or calculate flows. Flow pictures represented by streamlines are not invariant with respect to the Galilean transformation. Vortex structures can be absent in a certain system and well defined in another. It follows that the uniform motion of a coordinate system can mask the vortex structure which, in reality, exists.

Taking this into consideration, Jeong and Hussian developed a method for distinguishing space regions $\lambda_{2}(t, \boldsymbol{x})$ in which vortex structures can hypothetically exist [28]. The study performed in [28] showed that the $\lambda_{2}(t, x)$ regions were indeed capable of predicting the position of vortex structures in space. These predictions are, however, fairly approximate.

Namely, streamline flow pictures can contain regions with vortex structures that are undetectable by the JeongHussian method. Conversely, this method can show the regions of existence for vortex structures where, according to the streamline flow pictures, vortex structures are absent. Moreover, the Jeong-Hussian method outlines the $\lambda_{2}(t, \boldsymbol{x})$ contours of the supposed existence of vortex structures but does not indicate the particular coordinate system in which these vortex structures are to be sought. The Jeong-Hussian method cannot be used to correct flow pictures. On the contrary, the correctness of this method is evaluated by comparing the hypothetical regions where vortex structures with calculated flow pictures exist.

Once more, the Jeong-Hussian method [28] outlines only the boundaries of the $\lambda_{2}(t, \boldsymbol{x})$ regions. The boundaries of the $\lambda_{2}(t, \boldsymbol{x})$ regions are very widespread, because vortex structures from all the reference systems without exception must find a place within these $\lambda_{2}(t, x)$ regions. The method is incapable of penetrating the $\lambda_{2}(t, \boldsymbol{x})$ region. As a consequence, the method is incapable of revealing the presence or the absence of vortex structure within the $\lambda_{2}(t, \boldsymbol{x})$ region. Nevertheless, namely boundaries of the $\lambda_{2}(t, x)$ regions are put in correspondence to observed vortex structures. The ideas of appearance of vortex structures are based on the appearance of boundaries of the $\lambda_{2}(t, \boldsymbol{x})$ regions. The ideas of temporal evolution of vortex structures are based on temporal evolution of boundaries of the $\lambda_{2}(t, \boldsymbol{x})$ regions too.

The $\lambda_{2}(t, x)$ region of the supposed existence of vortex structures represented in Figure 31 from [20] extends in the wake behind a sphere to distances much larger than the sphere diameter. However, in the spherefixed $\operatorname{Ref}_{0}$ coordinate system the calculation does not find any vortex structures within the $\lambda_{2}(t, \boldsymbol{x})$ region (Figure 25 in [20]). To summarize, the $\lambda_{2}(t, \boldsymbol{x})$ region pattern does not remove the discrepancy between the streamline flow picture calculated in the $\operatorname{Ref}_{0}$ system of coordinates and the flow picture observed in the same system. Really, the experiment records the hairpin vortex shedding in the wake behind a sphere (Figure 7(a,i) in [7]). The streamline flow picture draws the periodic restructuring in the near wake, which causes wavy motion in the far wake without vortex shedding (Figure 25 in [20]).

There may exist such reference system $\operatorname{Ref}_{1}$ in which the vortex shedding appears in the calculated flow picture. Possibly, the flow picture calculated in the $\operatorname{Ref}_{1}$ system will be identical to the photographs of the flow obtained in the same $\operatorname{Ref}_{1}$ system. Today, calculation 
does not discover such reference system $\operatorname{Ref}_{1}$.

The possibilities of variation the boundary and initial conditions of calculations to bring it in strict correspondence to the experimental boundary and initial conditions are not very narrowly, the possibilities of perfecting the numerical procedure applied in recent simulations are boundless. Nevertheless, there are almost no prospects for a cardinal change in the calculated flow pictures [2]. It is therefore very improbable that the instability development direction calculated in $[15,16,18-21]$ can change and new solutions to the Navier-Stokes equations other than $\boldsymbol{U}_{s 0}^{\text {cal }}(\boldsymbol{x}), \boldsymbol{U}_{s 1}^{\text {cal }}(\boldsymbol{x}), \boldsymbol{U}_{s 2}^{c a l}(t, \boldsymbol{x})$, and $\boldsymbol{U}_{s 3}^{\text {cal }}(t, \boldsymbol{x})$ can appear.

It may well be that the interpretation of experimental results presented in Section 2 is not ultimate. Possibly, the directions presented will be augmented by new modes that have not been recorded up to now. It is also possible that the modes that have been recorded will be differently distributed over the three directions shown in Figure 1, and new modes will force us to modify the concepts upon which the three turbulence development directions are based. However, irrespective of any modifications of the scheme shown in Figure 1, it is very improbable that one of the conclusions drawn from comparing it with experiment will change. Namely, calculations are incapable of reproducing any of the six periodical vortex shedding modes observed along the three turbulence development directions (Figure 1). Most likely $[1,2]$, the reason for simulation failure is the NavierStokes equations themselves.

\section{Increase in the Number of Principal Hydrodynamic Values}

Classic hydrodynamics equations exist for about two centuries. By definition, these equations are valid for the description of arbitrary continuous media corresponding to continuity and unlimited deformability principles [29]. Statistically grounded hydrodynamics equations are, however, far from being completely established. The greatest progress in this direction was made for one of continuous medium states, namely, for the rarefied gas state, where the characteristic free path $\lambda$ far exceeded the characteristic size of particles $d$. In a rarefied gas, that is, at $d \ll \lambda$, the path from classic mechanics equations written separately for each of the $N$ medium particle to classic hydrodynamics equations was passed without additional assumptions. The only exception was the Boltzmann hypothesis of molecular chaos "Stosszahlansatz" (Figure 3).

Classic direction and pair-multimoment direction are two independent branches of the statistical deterministic approach to medium description. Classic kinetics and classic hydrodynamics are direct corollaries to the first equation of the Bogolyubov-Born-Green-Kirkwood-Yvon

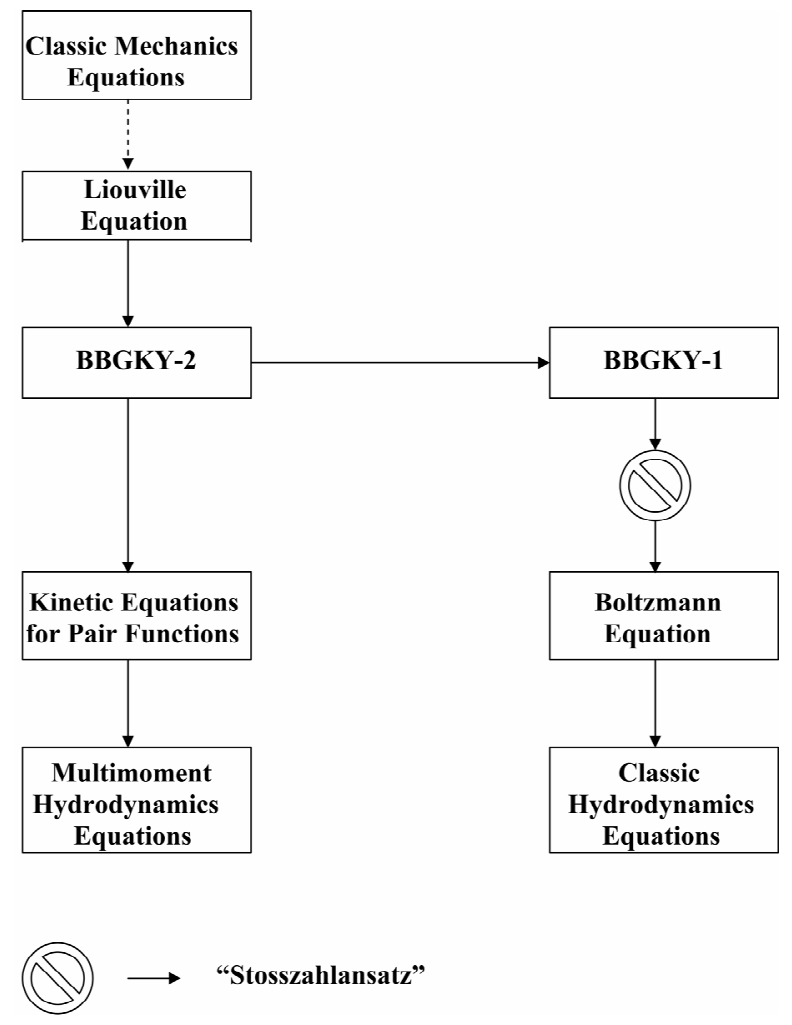

Figure 3. The scheme of the statistical deterministic approach.

chain of equations, i.e., the BBGKY hierarchy [30]. The kinetic equations for pair functions and the multimoment hydrodynamics equations are derived from the second of these equations. The BBGKY hierarchy is closed by the Liouville equation (Figure 3). To pass from the classical mechanics equations to the Liouville equation, the concept of the Gibbs ensemble is invoked. The Gibbs concept is the connecting link between the dynamical deterministic approach and the statistical deterministic approach [30].

The $s^{\text {th }}$ equation of the BBGKY hierarchy has the form,

$$
\begin{aligned}
& \left(\frac{\partial}{\partial t}+\sum_{i=1}^{s} \boldsymbol{\xi}_{i} \frac{\partial}{\partial \boldsymbol{x}_{i}}+\sum_{i=1}^{s} \sum_{j \neq i, j=1}^{s}\left(\frac{\boldsymbol{\Phi}_{i, j}}{m}\right) \frac{\partial}{\partial \boldsymbol{\xi}_{i}}\right) \\
& \cdot F_{s}\left(t, \boldsymbol{x}_{1}, \boldsymbol{\xi}_{1}, \boldsymbol{x}_{2}, \boldsymbol{\xi}_{2}, \cdots, \boldsymbol{x}_{s}, \boldsymbol{\xi}_{s}\right) \\
& =-(N-s) \sum_{i=1}^{s} \int\left(\frac{\boldsymbol{\Phi}_{i, s+1}}{m}\right) \frac{\partial}{\partial \boldsymbol{\xi}_{i}} \\
& \quad \cdot F_{s+1}\left(t, \boldsymbol{x}_{1}, \boldsymbol{\xi}_{1}, \boldsymbol{x}_{2}, \boldsymbol{\xi}_{2}, \cdots, \boldsymbol{x}_{s+1}, \boldsymbol{\xi}_{s+1}\right) \mathrm{d} \boldsymbol{x}_{s+1} \mathrm{~d} \boldsymbol{\xi}_{s+1}
\end{aligned}
$$

where $m$ is the mass of the particle; $\boldsymbol{\Phi}_{i, j}$ is the force of the action of the $i^{\text {th }}$ on $j^{\text {th }}$ particle; and $\boldsymbol{x}_{i}, \boldsymbol{\xi}_{i}$ are the spatial coordinate and velocity of the $i^{\text {th }}$ particle, respectively. The $F_{s}\left(t, \boldsymbol{x}_{1}, \boldsymbol{\xi}_{1}, \cdots, \boldsymbol{x}_{s}, \boldsymbol{\xi}_{s}\right)$ s-particle distribution function has the meaning of the probability that at time $t$ particle 1 finds itself in unit element of the phase 
space near point $\boldsymbol{x}_{i}, \boldsymbol{\xi}_{i}, \cdots$, and particle s near point $\boldsymbol{x}_{s}$, $\boldsymbol{\xi}_{s}$. The $F_{1}\left(t, \boldsymbol{x}_{1}, \boldsymbol{\xi}_{1}\right)$ one-particle distribution function obeys the first equation of the BBGKY hierarchy (1). The first equation is not closed. The term on the right hand side of Equation (1) that contains the

$F_{2}\left(t, \boldsymbol{x}_{1}, \boldsymbol{\xi}_{1}, \boldsymbol{x}_{2}, \boldsymbol{\xi}_{2}\right)$ two-particle function is responsible for the interaction of particle 1 with some particle 2 .

There are several variants of the derivation of the Boltzmann equation directly from the first equation of the BBGKY hierarchy [30]. Each of them reaches the equation,

$$
\begin{aligned}
& \left(\frac{\partial}{\partial t}+\boldsymbol{\xi}_{1} \frac{\partial}{\partial \boldsymbol{x}_{1}}\right) f_{1}\left(t, \boldsymbol{x}_{1}, \boldsymbol{\xi}_{1}\right)=N(N-1) J\left(t, \boldsymbol{x}_{1}, \boldsymbol{\xi}_{1}\right) \\
& J\left(t, \boldsymbol{x}_{1}, \boldsymbol{\xi}_{1}\right)=\int J\left(t, \boldsymbol{x}_{1}, \boldsymbol{\xi}_{1}, \boldsymbol{\xi}_{2}\right) \mathrm{d} \boldsymbol{\xi}_{2} \\
& J\left(t, \boldsymbol{x}_{1}, \boldsymbol{\xi}_{1}, \boldsymbol{\xi}_{2}\right)=\int v\left[\bar{F}_{2}\left(t, \boldsymbol{x}_{1}, \boldsymbol{\xi}_{1}^{\prime}, \boldsymbol{x}_{1}-\boldsymbol{\rho}_{\boldsymbol{v}^{\prime}}^{-d}, \boldsymbol{\xi}_{2}^{\prime}\right)\right. \\
& \left.-\bar{F}_{2}\left(t, \boldsymbol{x}_{1}, \boldsymbol{\xi}_{1}, \boldsymbol{x}_{1}-\boldsymbol{\rho}_{\boldsymbol{v}}^{-d}, \boldsymbol{\xi}_{2}\right)\right] b \mathrm{~d} b \mathrm{~d} \varepsilon
\end{aligned}
$$

Equation (2) is valid for a rarefied gas, that is, at $d \ll \lambda$,

$$
\begin{aligned}
& \bar{F}_{1}\left(t, \boldsymbol{x}_{1}, \boldsymbol{\xi}_{1}\right)=\frac{1}{l^{3}} \int_{W} F_{1}\left(t, \boldsymbol{x}_{1}+\boldsymbol{a}, \boldsymbol{\xi}_{1}\right) \mathrm{d} \boldsymbol{a} \\
& f_{1}\left(t, \boldsymbol{x}_{1}, \boldsymbol{\xi}_{1}\right)=N \bar{F}_{1}\left(t, \boldsymbol{x}_{1}, \boldsymbol{\xi}_{1}\right) \\
& \bar{F}_{2}\left(t, \boldsymbol{x}_{1}, \boldsymbol{\xi}_{1}, \boldsymbol{x}_{1}-\boldsymbol{\rho}, \boldsymbol{\xi}_{2}\right) \\
& =\frac{1}{l^{3}} \int_{W} F_{2}\left(t, \boldsymbol{x}_{1}+\boldsymbol{a}, \boldsymbol{\xi}_{1}, \boldsymbol{x}_{1}+\boldsymbol{a}-\boldsymbol{\rho}, \boldsymbol{\xi}_{2}\right) \mathrm{d} \boldsymbol{a}
\end{aligned}
$$

The one-particle distribution function $f_{1}\left(t, \boldsymbol{x}_{1}, \boldsymbol{\xi}_{1}\right)$ in Equations (2), (3) has the meaning of the probable number of particles situated at time $t$ in unit volume element near point $\boldsymbol{x}_{1}$ and having velocities in unit interval near the $\xi_{1}$ point. The spatial integration in Equation (3) is performed within the $W$ region having the characteristic linear size $l, d \ll l \ll \lambda$. The averaged $\bar{F}_{1}\left(t, \boldsymbol{x}_{1}, \boldsymbol{\xi}_{1}\right)$ and $\bar{F}_{2}\left(t, \boldsymbol{x}_{1}, \boldsymbol{\xi}_{1}, \boldsymbol{x}_{1}-\boldsymbol{\rho}, \boldsymbol{\xi}_{2}\right)$ functions weakly change on the scale of particle size $d$.

The $\bar{F}_{2}\left(t, \boldsymbol{x}_{1}, \boldsymbol{\xi}_{1}, \boldsymbol{x}_{1}-\boldsymbol{\rho}_{\boldsymbol{v}}^{-d}, \boldsymbol{\xi}_{2}\right)$ function on the right hand side of Equation (2) corresponds to a pair of particles 1 and 2, which enter the region of their interaction $C_{0}$ at velocities $\boldsymbol{\xi}_{1}$ and $\boldsymbol{\xi}_{2}$, respectively; $\boldsymbol{\rho}=\boldsymbol{x}_{1}-\boldsymbol{x}_{2}$, and $\boldsymbol{v}=\boldsymbol{\xi}_{1}-\boldsymbol{\xi}_{2}$ (Figure 4). The $\boldsymbol{\rho}_{\boldsymbol{v}}^{-d}$ vector has the coordinates $b, \varepsilon,-d$ in the cylindrical system of coordinates with the $z$ axis parallel to the $v$ vector, $b$ is the impact parameter, and $\varepsilon$ is the azimuthal angle. That is, the second term on the right hand side of Equation (2) is responsible for a decrease in the number of $\boldsymbol{\xi}_{1}$-particles in collisions with other particles. The

$\bar{F}_{2}\left(t, \boldsymbol{x}_{1}, \boldsymbol{\xi}_{1}^{\prime}, \boldsymbol{x}_{1}-\boldsymbol{\rho}_{\boldsymbol{v}^{\prime}}^{-d}, \boldsymbol{\xi}_{2}^{\prime}\right)$ function corresponds to a pair of particles 1 and 2, which enter the region of their interaction $C_{0}$ at velocities $\xi_{1}^{\prime}$ and $\boldsymbol{\xi}_{2}^{\prime}$, respectively, $\boldsymbol{v}^{\prime}=\boldsymbol{\xi}_{1}^{\prime}-\boldsymbol{\xi}_{2}^{\prime}$. The coordinates of the $\boldsymbol{\rho}_{\boldsymbol{v}^{\prime}}^{-d}$ vector are

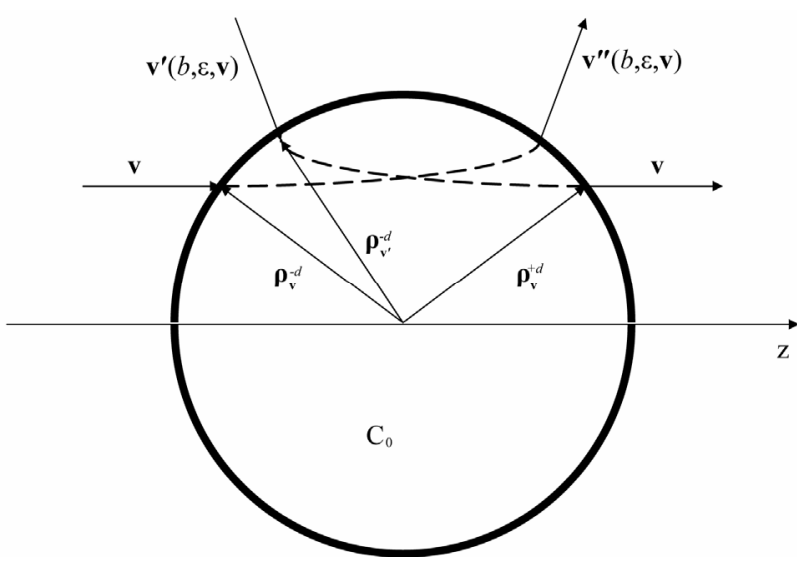

Figure 4. Interaction region $C_{0}$ of a pair of particles with characteristic radius $d$. If, at the entrance of region $C_{0}$, particles have velocity $v$ and parameters $b, \varepsilon$, they acquire velocity $v^{\prime \prime}(b, \varepsilon, v)$ at its exit. If, at the entrance of region $\mathrm{C}_{0}$, particles have relative movement velocity $\mathrm{v}^{\prime}(b, \varepsilon, v)$ and collision parameters $b, \varepsilon$, they acquire velocity $v$ at its exit.

$b, \varepsilon,-d$ in the cylindrical system coordinates with the $\mathrm{Z}$ axis parallel to $\boldsymbol{v}^{\prime}$. At the exit of the region of their interaction, particles 1 and 2 then have velocities $\xi_{1}$ and $\boldsymbol{\xi}_{2}$ respectively (Figure 4 ), that is, the first term on the right hand side of (2) is responsible for an increase in the number of $\boldsymbol{\xi}_{1}$-particles caused by collisions.

The factorization of two-particle distribution functions in $J\left(t, \boldsymbol{x}_{1}, \boldsymbol{\xi}_{1}, \boldsymbol{\xi}_{2}\right)$ from Equation (2), that is, their representation in the form of the product of two one-particle functions,

$$
\begin{aligned}
& \bar{F}_{2}\left(t, \boldsymbol{x}_{1}, \boldsymbol{\xi}_{1}, \boldsymbol{x}_{1}-\boldsymbol{\rho}_{v}^{-d}, \boldsymbol{\xi}_{2}\right)=\bar{F}_{1}\left(t, \boldsymbol{x}_{1}, \boldsymbol{\xi}_{1}\right) \bar{F}_{1}\left(t, \boldsymbol{x}_{1}, \boldsymbol{\xi}_{2}\right) \\
& \bar{F}_{2}\left(t, \boldsymbol{x}_{1}, \boldsymbol{\xi}_{1}^{\prime}, \boldsymbol{x}_{1}-\boldsymbol{\rho}_{v^{\prime}}^{-d}, \boldsymbol{\xi}_{2}^{\prime}\right)=\bar{F}_{1}\left(t, \boldsymbol{x}_{1}, \boldsymbol{\xi}_{1}^{\prime}\right) \bar{F}_{1}\left(t, \boldsymbol{x}_{1}, \boldsymbol{\xi}_{2}^{\prime}\right)
\end{aligned}
$$

leads to a closed Boltzmann classic kinetic equation,

$$
\begin{aligned}
& \left(\frac{\partial}{\partial t}+\boldsymbol{\xi}_{1} \frac{\partial}{\partial \boldsymbol{x}_{1}}\right) f_{1}\left(t, \boldsymbol{x}_{1}, \boldsymbol{\xi}_{1}\right) \\
& =\int v\left[f_{1}\left(t, \boldsymbol{x}_{1}, \boldsymbol{\xi}_{1}^{\prime}\right) f_{1}\left(t, \boldsymbol{x}_{1}, \boldsymbol{\xi}_{2}^{\prime}\right)-f_{1}\left(t, \boldsymbol{x}_{1}, \boldsymbol{\xi}_{1}\right) f_{1}\left(t, \boldsymbol{x}_{1}, \boldsymbol{\xi}_{2}\right)\right] \\
& \cdot b \mathrm{~d} b \mathrm{~d} \varepsilon \mathrm{d} \boldsymbol{\xi}_{2}
\end{aligned}
$$

The approximation that we use was called the molecular chaos hypothesis "Stosszahlansatz" [31]. Classic hydrodynamics equations follow directly from the Boltzmann equation (Figure 3) and, naturally, include errors made in the derivation of the classic kinetic equation. Let us elucidate the physical meaning of the error introduced by the Boltzmann hypothesis into hydrodynamics.

Let us pass in Equation (2) from $\bar{F}_{2}$ functions written in and $\boldsymbol{x}_{1}, \boldsymbol{\xi}_{1}, \boldsymbol{x}_{2}, \boldsymbol{\xi}_{2}$ variables to $\overline{\tilde{F}}_{2}$ functions written in $\boldsymbol{x}=\left(\boldsymbol{x}_{1}+\boldsymbol{x}_{2}\right) / 2, \boldsymbol{G}=\left(\boldsymbol{\xi}_{1}+\boldsymbol{\xi}_{2}\right) / 2, \boldsymbol{\rho}$, and $\boldsymbol{v}$ vari- 
ables,

$$
\begin{aligned}
& \tilde{J}\left(t, \boldsymbol{x}_{1}, \boldsymbol{G}, \boldsymbol{v}\right) \\
& =\int v\left[\tilde{F}_{2}\left(t, \boldsymbol{x}_{1}, \boldsymbol{G}, \boldsymbol{\rho}_{\boldsymbol{v}^{\prime}}^{-d}, \boldsymbol{v}^{\prime}\right)-\overline{\tilde{F}}_{2}\left(t, \boldsymbol{x}_{1}, \boldsymbol{G}, \boldsymbol{\rho}_{v}^{-d}, \boldsymbol{v}\right)\right] b \mathrm{~d} b \mathrm{~d} \varepsilon \\
& \tilde{J}\left(t, \boldsymbol{x}_{1}, \boldsymbol{G}, \boldsymbol{v}\right)=J\left(t, \boldsymbol{x}_{1}, \boldsymbol{\xi}_{1}, \boldsymbol{\xi}_{2}\right) \\
& F_{2}\left(t, \boldsymbol{x}_{1}, \boldsymbol{\xi}_{1}, \boldsymbol{x}_{2}, \boldsymbol{\xi}_{2}\right)=\tilde{F}_{2}(t, \boldsymbol{x}, \boldsymbol{G}, \boldsymbol{\rho}, \boldsymbol{v})
\end{aligned}
$$

The velocity of the center of mass of pair particles, $\boldsymbol{G}$, and the modulus $v$ of the relative velocity of particles $v=|v|$, are invariants of a binary particle collision. The enumeration of all the admissible target parameter values $b$ and $\varepsilon$ and relative motion velocity directions $\boldsymbol{v}$ at fixed $\boldsymbol{G}$ and $v$ values then gives all the possible velocity directions $v^{\prime}$. It follows that a collision-caused decrease in the number of pairs of particles from a unit phase volume interval near the $\boldsymbol{x}_{1}, \boldsymbol{G}, \boldsymbol{v}$ point characterized by all the admissible $b$ and $\varepsilon$ parameter values and relative motion $\boldsymbol{v}$ orientations is strictly balanced by a collision induced increase in the number of pairs of particles in this interval with these parameters,

$$
\int \tilde{J}\left(t, \boldsymbol{x}_{1}, \boldsymbol{G}, \boldsymbol{v}\right) \mathrm{d}^{2} \Omega=0
$$

Here $\mathrm{d}^{2} \Omega=\sin \theta \mathrm{d} \theta \mathrm{d} \varphi$, and $\theta$ and $\varphi$ are the spherical coordinates of the $v$ vector.

Suppose that $\varphi_{2}^{(n)}(\boldsymbol{G}, v), n=0,1, \cdots$, is an arbitrary weight function of velocities $\boldsymbol{G}$ and $v$. The properties of the $\varphi_{2}^{(n)}(\boldsymbol{G}, v), n=0,1, \cdots$, pair are invariants of a particle binary collision. Let us multiply the

$\tilde{J}\left(t, \boldsymbol{x}_{1}, \boldsymbol{G}, \boldsymbol{v}\right)$ collision integral determined by Equation (6) by $\varphi_{2}^{(n)}(\boldsymbol{G}, v), n=0,1, \cdots$, and integrate the result with respect to velocities. By virtue of Equation (7), we then have,

$$
\int \varphi_{2}^{(n)}(\boldsymbol{G}, v) \tilde{J}\left(t, \boldsymbol{x}_{1}, \boldsymbol{G}, \boldsymbol{v}\right) \mathrm{d} \boldsymbol{G} \mathrm{d} \boldsymbol{v}=0
$$

This means that particle collisions cannot influence the formation of hydrodynamic values constructed on the properties of the $\varphi_{2}^{(n)}(\boldsymbol{G}, v), n=0,1, \cdots$, pair. In other words, particle collisions cannot tune the distributions of all these hydrodynamic values to distributions of some other hydrodynamic values. That is, the set of hydrodynamic values constructed on the property of $\varphi_{2}^{(n)}(\boldsymbol{G}, v)$, $n=0,1, \cdots$,

$$
\begin{aligned}
& M^{(n)}\left(t, \boldsymbol{x}_{1}\right) \\
& =\frac{1}{A\left(t, \boldsymbol{x}_{1}\right)} \int \varphi_{2}^{(n)}(\boldsymbol{G}, v) v \overline{\tilde{F}}_{2}\left(t, \boldsymbol{x}_{1}, \boldsymbol{G}, \boldsymbol{\rho}_{\boldsymbol{v}}^{-d}, \boldsymbol{v}\right) b \mathrm{~d} b \mathrm{~d} \varepsilon \mathrm{d} \boldsymbol{G} \mathrm{d} \boldsymbol{v}
\end{aligned}
$$

is a set of the principal hydrodynamic values, the $A\left(t, \boldsymbol{x}_{1}\right)$ is local proportionality coefficient. Set (9) contains not only the lower $\overline{\tilde{F}}_{2}$ function moments but also all the higher moments without exception. To summarize, our analysis of the properties of the $\tilde{J}\left(t, \boldsymbol{x}_{1}, \boldsymbol{G}, \boldsymbol{v}\right)$ colli- sion integral reveals the existence of an infinite number of principal hydrodynamic values.

Under free molecular conditions, when $\lambda \gg L$, particle collisions do not participate in the formation of distributions of hydrodynamic values. These distributions are formed under the influence of the initial and boundary problem conditions, that is, each hydrodynamic value is principal. As the density of medium increases, the influence of intermolecular collisions on the formation of hydrodynamic value distributions increases. In a continuous medium, $\lambda \ll L$, this influence becomes predominant. Namely, binary particle collisions tune the distributions of all the function moments to distribution of moments (9). The velocities $\boldsymbol{G}$ and $v$ are invariants of a particle binary collision. That is why, according to Equation (8), moments (9), as previously, do not experience the influence of binary particle collisions. As in the free molecular mode, that is, at $\lambda \ll L$, moments (9) remain principal hydrodynamic values.

The equation for the $f_{1}\left(t, \boldsymbol{x}_{1}, \boldsymbol{\xi}_{1}\right)$ one-particle distribution function is written in a six-dimensional phase space of one particle ( $\mu$ space). The dimensionality of the $\mu$ space allows only the properties of particle $\varphi_{1}^{(n)}\left(\xi_{1}\right), n=0,1, \cdots$, to be accommodated in it; binary particle collision invariants, that is, $\varphi_{2}^{(n)}(\boldsymbol{G}, v)$, $n=0,1, \cdots$, do not fit into the $\mu$ space. The symmetry properties of the $J\left(t, \boldsymbol{x}_{1}, \boldsymbol{\xi}_{1}, \boldsymbol{\xi}_{2}\right)$ collision integral strictly correspond to the symmetry properties of the Boltzmann collision integral (5), [31]. It follows that only three lower particle properties, namely $1, \xi_{1}, \xi_{1}^{2}$, can lead to the disappearance of the $J\left(t, \boldsymbol{x}_{1}, \boldsymbol{\xi}_{1}\right)$ collision integral. Let us sequentially accommodate three lower properties of a particle in the $\mu$ space. We then have,

$$
\begin{aligned}
0 & =\int J\left(t, \boldsymbol{x}_{1}, \boldsymbol{\xi}_{1}\right) \mathrm{d} \boldsymbol{\xi}_{1}=\int \tilde{J}\left(t, \boldsymbol{x}_{1}, \boldsymbol{G}, \boldsymbol{v}\right) \mathrm{d} \boldsymbol{G} \mathrm{d} \boldsymbol{v} \\
0 & =\int \boldsymbol{\xi}_{1} J\left(t, \boldsymbol{x}_{1}, \boldsymbol{\xi}_{1}\right) \mathrm{d} \xi_{1} \\
& =\int\left(\boldsymbol{G}+\frac{\boldsymbol{v}}{2}\right) \tilde{J}\left(t, \boldsymbol{x}_{1}, \boldsymbol{G}, \boldsymbol{v}\right) \mathrm{d} \boldsymbol{G} \mathrm{d} \boldsymbol{v} \\
& =\int \boldsymbol{G} \tilde{J}\left(t, \boldsymbol{x}_{1}, \boldsymbol{G}, \boldsymbol{v}\right) \mathrm{d} \boldsymbol{G} \mathrm{d} \boldsymbol{v} \\
0 & =\int \xi_{1}^{2} J\left(t, \boldsymbol{x}_{1}, \boldsymbol{\xi}_{1}\right) \mathrm{d} \boldsymbol{\xi}_{1} \\
& =\int\left(\boldsymbol{G}^{2}+\frac{\boldsymbol{v}^{2}}{4}+\boldsymbol{G} \boldsymbol{v}\right) \tilde{J}\left(t, \boldsymbol{x}_{1}, \boldsymbol{G}, \boldsymbol{v}\right) \mathrm{d} \boldsymbol{G} \mathrm{d} \boldsymbol{v} \\
& =\int\left(\boldsymbol{G}^{2}+\frac{\boldsymbol{v}^{2}}{4}\right) \tilde{J}\left(t, \boldsymbol{x}_{1}, \boldsymbol{G}, \boldsymbol{v}\right) \mathrm{d} \boldsymbol{G} \mathrm{d} \boldsymbol{v}
\end{aligned}
$$

Equations (10) are valid because of symmetry of the partial distribution function $\tilde{F}_{2}(t, \boldsymbol{x}, \boldsymbol{G}, \boldsymbol{\rho}, \boldsymbol{v})$ with respect to the permutation of the phase coordinates of two particles [3]. The symmetry properties allow all the moments of the two-particle distribution functions odd with respect to $v$ to be removed from (10), [32]. 
It follows that the integrals that contain the lower particle properties $1, \xi_{1}, \xi_{1}^{2}$, as weight functions are strictly reduced to the integrals with the $1, G,\left(G^{2}+v^{2} / 4\right)$ weight functions belonging to $\varphi_{2}^{(n)}(\boldsymbol{G}, v) n=0,1, \cdots$, invariants. Three lower particle properties $1, \boldsymbol{\xi}_{1}$, and $\boldsymbol{\xi}_{1}^{2}$ give three lower $f_{1}\left(t, \boldsymbol{x}_{1}, \boldsymbol{\xi}_{1}\right)$ one-particle distribution function moments, the density of the number of particles $n(t, \boldsymbol{x})$, medium velocity $\boldsymbol{U}(t, \boldsymbol{x})$, and medium pressure $p(t, \boldsymbol{x})$. It follows that, when we pass to the hydrodynamic stage from the phase space of one particle, such hydrodynamics equations cannot be constructed using more than three lower principal hydrodynamic values corresponding to the $1, \xi_{1}, \xi_{1}^{2}$ particle properties. The transition to the hydrodynamic stage from the phase space of one particle excludes higher principal hydrodynamic values (9) from participation in the construction of hydrodynamics equations. However, there is no rigorous passage to hydrodynamics from the $\mu$ space. This passage is closed because Equation (2) is not closed.

The use of the Boltzmann hypothesis ("Stosszahlansatz") (4) opens up the possibility of approximate passage to hydrodynamics. So, the physical meaning of the error introduced by the Boltzmann hypothesis into hydrodynamics is as follows. It follows that just Boltzmann hypothesis allows us to construct hydrodynamics on only three lower principal hydrodynamic values. It follows that the use of the Boltzmann hypothesis excludes higher principal hydrodynamic values (9) from the participation in the formation of classic hydrodynamics equations. Since the classic three-moment hydrodynamics is constructed without the use of values (9), its applicability range is limited to states that are only weakly removed from the statistical equilibrium state. To substantiate this conclusion, we must find passage to hydrodynamics from the phase space capable of accommodateing the whole set of binary particle collision $\varphi_{2}^{(n)}(\boldsymbol{G}, v)$ $n=0,1, \cdots$, invariants.

The $F_{2}\left(t, \boldsymbol{x}_{1}, \boldsymbol{\xi}_{1}, \boldsymbol{x}_{2}, \boldsymbol{\xi}_{2}\right)$ two-particle distribution function obeys the second equation of the BBGKY hierarchy (1). The second equation is valid for arbitrary positions of particles 1 and 2 in space with respect to each other. It is not closed. There are, however, positions of particles 1 and 2 for which the second equation is considerably simplified.

The left hand side of the second equation of the BBGKY hierarchy (1),

$$
\begin{aligned}
& {\left[\frac{\partial}{\partial t}+\boldsymbol{\xi}_{1} \frac{\partial}{\partial \boldsymbol{x}_{1}}+\boldsymbol{\xi}_{2} \frac{\partial}{\partial \boldsymbol{x}_{2}}+\left(\frac{\boldsymbol{\Phi}_{1,2}}{m}\right) \frac{\partial}{\partial \boldsymbol{\xi}_{1}}+\left(\frac{\boldsymbol{\Phi}_{2,1}}{m}\right) \frac{\partial}{\partial \boldsymbol{\xi}_{2}}\right]} \\
& \cdot F_{2}\left(t, \boldsymbol{x}_{1}, \boldsymbol{\xi}_{1}, \boldsymbol{x}_{2}, \boldsymbol{\xi}_{2}\right) \\
& =-(N-2) \int\left[\left(\frac{\boldsymbol{\Phi}_{1,3}}{m}\right) \frac{\partial}{\partial \boldsymbol{\xi}_{1}}+\left(\frac{\boldsymbol{\Phi}_{2,3}}{m}\right) \frac{\partial}{\partial \boldsymbol{\xi}_{2}}\right] \\
& \cdot F_{3}\left(t, \boldsymbol{x}_{1}, \boldsymbol{\xi}_{1}, \boldsymbol{x}_{2}, \boldsymbol{\xi}_{2}, \boldsymbol{x}_{3}, \boldsymbol{\xi}_{3}\right) \mathrm{d} \boldsymbol{x}_{3} \mathrm{~d} \boldsymbol{\xi}_{3}
\end{aligned}
$$

describes the free movement of particles 1 and 2 and the interaction between the particles 1 and 2. The integral terms containing the $F_{3}\left(t, \boldsymbol{x}_{1}, \boldsymbol{\xi}_{1}, \boldsymbol{x}_{2}, \boldsymbol{\xi}_{2}, \boldsymbol{x}_{3}, \boldsymbol{\xi}_{3}\right)$ threeparticle distribution function are responsible for the interaction of particles 1 and 2 with some third particle 3 . Presuming that a particle may be present at all phase space locations with equal probabilities, introduce the dimensionless quantities,

$$
\begin{aligned}
& F_{2}=\left(1 / V^{2} c^{6}\right) \hat{F}_{2}, \quad F_{3}=\left(1 / V^{3} c^{9}\right) \hat{F}_{3}, \\
& \boldsymbol{\xi}_{i}=c \hat{\boldsymbol{\xi}}_{i}, \quad\left(\frac{\boldsymbol{\Phi}_{i, j}}{m}\right)=\left(c^{2} / d\right)\left(\frac{\hat{\boldsymbol{\Phi}}_{i, j}}{\hat{m}}\right), \quad i, j=1,2,3
\end{aligned}
$$

In Equation (12) the hat appears above the dimensionless quantities, $c$ is the characteristic velocity of the particle, the ratio of the system volume $V$ to the characteristic volume $d^{2} \lambda$ occupied by one particle is used as an estimation for $N$. In the thermodynamic limit, $N \rightarrow \infty, V \rightarrow \infty$, yet, $N / V$ is finite.

Let us bring the convective terms in the left hand side of Equation (11) into dimensionless form on the scale of particle size, $t=(d / c) \hat{t}, \boldsymbol{x}_{i}=d \hat{\boldsymbol{x}}_{i}$. In this case, the ratio of order of the right hand side terms to order of the left hand side terms of Equation (11) is of the order of $d / \lambda, d \ll \lambda$. It means that the interaction with third particle has a very weak influence on the free movement of particles 1 and 2 and the interaction between the particles 1 and 2 at times, proportional to $d / c$. The right hand side terms of Equation (11) receive the fundamental order only at times, proportional to $\lambda / c$.

Let us substitute the force term of the left hand side of the third equation of the BBGKY hierarchy (1) into the first term of the right hand side of Equation (11). Then,

$$
\begin{array}{r}
-(N-2) \int\left(\frac{\boldsymbol{\Phi}_{1,3}}{m}\right) \frac{\partial}{\partial \boldsymbol{\xi}_{1}} F_{3}\left(t, \boldsymbol{x}_{1}, \boldsymbol{\xi}_{1}, \boldsymbol{x}_{2}, \boldsymbol{\xi}_{2}, \boldsymbol{x}_{3}, \boldsymbol{\xi}_{3}\right) \mathrm{d} \boldsymbol{x}_{3} \mathrm{~d} \boldsymbol{\xi}_{3} \\
=(N-2) \int w\left[\tilde{\tilde{F}}_{3}\left(t, \boldsymbol{x}_{1}, \boldsymbol{\xi}_{1}, \boldsymbol{x}_{2}, \boldsymbol{\xi}_{2}, \boldsymbol{r}_{\boldsymbol{w}}^{+d}, \boldsymbol{w}\right)\right. \\
\left.-\tilde{\tilde{F}}_{3}\left(t, \boldsymbol{x}_{1}, \boldsymbol{\xi}_{1}, \boldsymbol{x}_{2}, \boldsymbol{\xi}_{2}, \boldsymbol{r}_{\boldsymbol{w}}^{-d}, \boldsymbol{w}\right)\right] b \mathrm{~d} b \mathrm{~d} \varepsilon \mathrm{d} \boldsymbol{w}
\end{array}
$$

Here,

$\tilde{\tilde{F}}_{3}\left(t, \boldsymbol{x}_{1}, \boldsymbol{\xi}_{1}, \boldsymbol{x}_{2}, \boldsymbol{\xi}_{2}, \boldsymbol{r}, \boldsymbol{w}\right)=F_{3}\left(t, \boldsymbol{x}_{1}, \boldsymbol{\xi}_{1}, \boldsymbol{x}_{2}, \boldsymbol{\xi}_{2}, \boldsymbol{x}_{3}, \boldsymbol{\xi}_{3}\right)$, $\boldsymbol{r}=\boldsymbol{x}_{1}-\boldsymbol{x}_{3}, \boldsymbol{w}=\xi_{1}-\xi_{3}$. In the right hand side of Equation (13), the terms responsible for triple collisions of particles are omitted. The convective terms responsible for displacement of particles 1 and 2 during the time of interaction of particles 1 with particle 3 are omitted too. The omitted terms are negligible with respect to saved terms in the right hand side of Equation (13). The estimation of orders is carried out in accordance with Equation (12). As noted above, the order of the convective terms is estimated at times, proportional to $\lambda / c$. 
Positions of particles 1 and 3, described by the first and second terms of the right hand side of Equation (13), are depicted in Figure 4. However, when interpreting the collision integral (13) the vectors $\boldsymbol{v}, \boldsymbol{\rho}_{v}^{+d}, \rho_{v}^{-d}$ in Figure 4, should be replaced respectively by the vectors $\boldsymbol{w}, \boldsymbol{r}_{w}^{+d}, \boldsymbol{r}_{w}^{-d}$. The first term of the right hand side of Equation (13) is responsible for an increase in the number of $\xi_{1}$-particles caused by collisions with some third $\boldsymbol{\xi}_{3}$-particles $\left(\boldsymbol{r}=\boldsymbol{r}_{w}^{+d}\right)$. If at time $t$ particles 1 and 3 leave the region of their interaction $C_{0}$, then later, within a time interval not exceeding $\lambda / c$, they will be placed with respect to each other within the cylindrical volume oriented in the direction of vector $\boldsymbol{w}$ with bases area of $\pi d^{2}$ and height of $\lambda$. I.e., these particles within a time interval of $\lambda / c$ will be in the stage of departure from each other. The second term of the right hand side of Equation (13) is responsible for a decrease in the number of $\xi_{1}$-particles in collisions with some third $\boldsymbol{\xi}_{3}$-particles $\left(\boldsymbol{r}=\boldsymbol{r}_{w}^{-d}\right)$. If at time $t$ particles 1 and 3 enter the region of their interaction $C_{0}$, then earlier, within a time interval not exceeding $\lambda / c$, they were placed with respect to each other within the cylindrical volume oriented in the direction of vector $\boldsymbol{w}$ with bases area of $\pi d^{2}$ and height of $\lambda$. i.e., these particles within a time interval of $\lambda / c$ were in the stage of approaching with each other. Thus, the terms of the right hand side of Equation (13) at times, proportional to $\lambda / c$, describe groups of three particles, in which two of three particles (1 and 3) find itself either at the stage of approaching before the collision with each other, or in the stage of departure from each other after collision. The second term of the right hand side of Equation (11) has the identical physical meaning.

The idea that leads to the concept of pair distribution functions is as follows. For instance, in a rarefied gas at each time moment, each particle after its last collision moves toward the next collision. This means that every particle 1 in a rarefied gas simultaneously flies away from some particle 2 with which it collided last at point $D$ (Figure 5), and approaches some particle 2 with which it is to collide next at point $B$.

It follows that it is particle 2 plays the role of a third particle 3 for particle 1 , with which particle 1 has already experienced a collision and will be in the stage of departure within a time interval not exceeding $\lambda / c$ (Figure 5(a)). At the same time, it is particle 1 plays the role of a third particle 3 for particle 2, with which the particle 2 has already experienced a collision and will be in the stage of departure within a time interval not exceeding $\lambda / c$ (Figure 5(a)). From the other hand, it is a particle 2 plays the role of a third particle 3 for particle 1, with which particle 1 should collide within a time interval not exceeding $\lambda / c$ (Figure 5(b)). At the same time, it is particle 1 plays the role of a third particle 3 for particle 2 ,

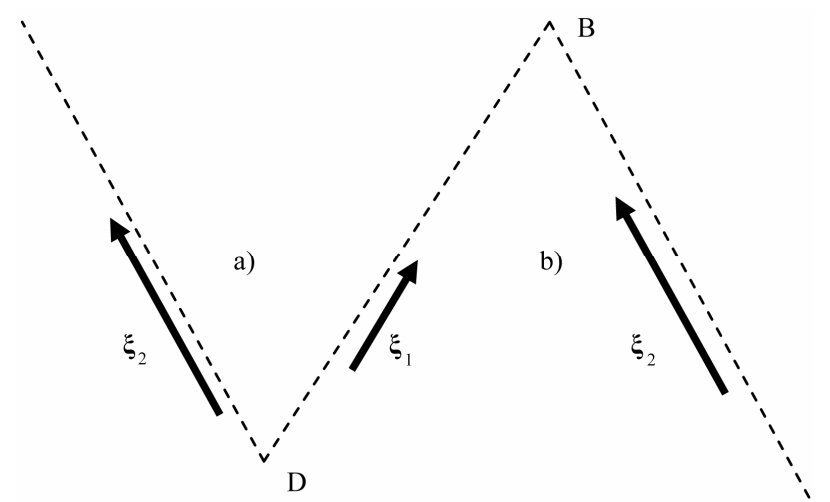

Figure 5. Graphic representation of a pair of particles.

with which the particle 2 should collide within a time interval not exceeding $\lambda / c$ (Figure 5(b)).

The motion and interaction of particles 1 and 2 are, however, described by the left-hand side terms of the second equation of hierarchy (11) that are proportional to $F_{2}\left(t, \boldsymbol{x}_{1}, \boldsymbol{\xi}_{1}, \boldsymbol{x}_{2}, \boldsymbol{\xi}_{2}\right)$. For this reason, the terms containing $F_{3}\left(t, \boldsymbol{x}_{1}, \boldsymbol{\xi}_{1}, \boldsymbol{x}_{2}, \boldsymbol{\xi}_{2}, \boldsymbol{x}_{3}, \boldsymbol{\xi}_{3}\right)$ must be excluded for particles 1 and 2. The second equation of the BBGKY hierarchy (11) written for particles 1 and 2 (Figure 5), is therefore considerably simplified,

$$
\begin{aligned}
& \left(\frac{\partial}{\partial t}+\boldsymbol{\xi}_{1} \frac{\partial}{\partial \boldsymbol{x}_{1}}+\boldsymbol{\xi}_{2} \frac{\partial}{\partial \boldsymbol{x}_{2}}+\frac{\boldsymbol{\Phi}_{12}}{m} \frac{\partial}{\partial \boldsymbol{\xi}_{1}}+\frac{\boldsymbol{\Phi}_{21}}{m} \frac{\partial}{\partial \boldsymbol{\xi}_{2}}\right) \\
& \cdot F_{2}^{p}\left(t, \boldsymbol{x}_{1}, \boldsymbol{\xi}_{1}, \boldsymbol{x}_{2}, \boldsymbol{\xi}_{2}\right)=0
\end{aligned}
$$

Generally, any medium particle forms a pair with every other particle. A medium therefore contains $N(N-1)$ pairs of particles. All these pairs are described by the $F_{2}\left(t, \boldsymbol{x}_{1}, \boldsymbol{\xi}_{1}, \boldsymbol{x}_{2}, \boldsymbol{\xi}_{2}\right)$ function, which obeys the second equation of hierarchy (11). If a single particle 2 , which either flies away from (Figure 5(a)), or approaches (Figure 5(b)), some particle 1 , is selected as a partner of this particle, this pair is described by the $F_{2}^{p}\left(t, \boldsymbol{x}_{1}, \boldsymbol{\xi}_{1}, \boldsymbol{x}_{2}, \boldsymbol{\xi}_{2}\right)$ function that obeys Equation (14). Note that Equation (14) is valid for an arbitrary gas particle rather than some particular particle 1 (Figure 5). For this reason, Equation (14) is capable of describing the gas as a whole. A rigorous derivation of Equation (14) is given in [33]. There is an analogy between the derivation [33] and Grad method used in deriving the Boltzmann equation for a gas consisting of rigid spheres [30].

The arguments of the $F_{2}^{p}\left(t, \boldsymbol{x}_{1}, \boldsymbol{\xi}_{1}, \boldsymbol{x}_{2}, \boldsymbol{\xi}_{2}\right)$ function are $\boldsymbol{x}_{1}$ and $\boldsymbol{x}_{2}$, which are the spatial coordinates of particles 1 and 2. Information about the position of a separate particle in space, however, becomes lost in kinetics and hydrodynamics. Classic kinetics and hydrodynamics deal with the place in space near which a set of particles are situated rather than with particle coordinates. It follows that a necessary condition for the transition from (14) to kinetics and hydrodynamics equations is 
getting rid of one of the spatial coordinates of the $F_{2}^{p}$ function. It was suggested in [33] that $F_{2}^{p}$ be integrated in one of its spatial coordinates within the cylinder of collisions. The $\Delta_{1}$ and $\Delta_{2}$ cylinders of collisions are oriented in the direction of the velocity $v$, the areas of their bases equal the collision cross section area $\pi d^{2}$, and the heights of the cylinders equal the characteristic free path $\lambda(t, \boldsymbol{x})$. Let us pass from the 12-dimensional phase space of particles 1 and 2 constructed on the $\boldsymbol{x}_{1}, \boldsymbol{\xi}_{1}, \boldsymbol{x}_{2}, \boldsymbol{\xi}_{2}$ vectors to the 12 -dimensional space constructed on the $\boldsymbol{x}, \boldsymbol{G}, \boldsymbol{\rho}, \boldsymbol{v}$ vectors and produce pair distribution functions $f_{p}^{a p p}(t, \boldsymbol{x}, \boldsymbol{G}, \boldsymbol{v})$ and $f_{p}^{d i v}(t, \boldsymbol{x}, \boldsymbol{G}, \boldsymbol{v})$,

$$
\begin{aligned}
& f_{p}^{a p p}(t, \boldsymbol{x}, \boldsymbol{G}, \boldsymbol{v}) \\
& =\frac{N(N-1)}{2 l^{3}} \int_{W} \int_{\Delta_{1}} \tilde{F}_{2}(t, \boldsymbol{x}+\boldsymbol{a}, \boldsymbol{G}, \boldsymbol{\rho}, \boldsymbol{v}) \mathrm{d} \boldsymbol{\rho} \mathrm{d} \\
& f_{p}^{d i v}(t, \boldsymbol{x}, \boldsymbol{G}, \boldsymbol{v}) \\
& =\frac{N(N-1)}{2 l^{3}} \int_{W} \int_{\Delta_{2}} \tilde{F}_{2}(t, \boldsymbol{x}+\boldsymbol{a}, \boldsymbol{G}, \boldsymbol{\rho}, \boldsymbol{v}) \mathrm{d} \boldsymbol{\rho} \mathrm{d} \boldsymbol{a} \\
& F_{2}\left(t, \boldsymbol{x}_{1}, \boldsymbol{\xi}_{1}, \boldsymbol{x}_{2}, \boldsymbol{\xi}_{2}\right)=\tilde{F}_{2}(t, \boldsymbol{x}, \boldsymbol{G}, \boldsymbol{\rho}, \boldsymbol{v})
\end{aligned}
$$

Integration (15) collects all pairs of particles that either will inevitably collide during the time interval not exceeding the characteristic time between collisions $\tau=\lambda / v$ or fly away from each other after a collision that occurs during the time interval not exceeding $\tau$. The integration with respect to $\boldsymbol{a}$ within $W$ region removes strong spatial dependence of the functions on the scale having the characteristic size of particles $d$. The pair distribution function $f_{p}^{a p p}(t, \boldsymbol{x}, \boldsymbol{G}, \boldsymbol{v})$

$\left(f_{p}^{d i v}(t, \boldsymbol{x}, \boldsymbol{G}, \boldsymbol{v})\right)$ has the meaning of the probable number of pairs of particles approaching (diverging from) each other whose centers of mass are concentrated at time $t$ in a unit volume element near point $\boldsymbol{x}$ and the velocities of the centers of mass and relative particle velocities, in unit velocity elements near $\boldsymbol{G}$ and $\boldsymbol{v}$, respectively. The relations between pair distribution functions (15) and $v \overline{\tilde{F}}_{2}\left(t, \boldsymbol{x}_{1}, \boldsymbol{G}, \boldsymbol{\rho}_{\boldsymbol{v}}^{-d}, \boldsymbol{v}\right)$, and $f_{1}\left(t, \boldsymbol{x}_{1}, \boldsymbol{\xi}_{1}\right)$ from [3] can be used to find the proportionality coefficient (9),

$$
A=N(N-1) \tau
$$

Heuristic derivation of a system of kinetic equations for pair distribution functions $f_{p}^{a p p}(t, \boldsymbol{x}, \boldsymbol{G}, \boldsymbol{v})$ and $f_{p}^{d i v}(t, \boldsymbol{x}, \boldsymbol{G}, \boldsymbol{v})$ was given in [34]. In [33], equations for pair distribution functions (15) are derived directly from the fundamental statistical mechanics concepts. An analysis of the system of kinetic equations [33,34] reveals the most important property of $f_{p}(t, \boldsymbol{x}, \boldsymbol{G}, v)$ pair distribution function constructed on the velocity of the center of mass $\boldsymbol{G}$ and the relative velocity modulus $v$,

$$
\left(\frac{\partial}{\partial t}+\boldsymbol{G} \frac{\partial}{\partial \boldsymbol{x}}\right) f_{p}(t, \boldsymbol{x}, \boldsymbol{G}, v)=0
$$

Here,

$$
\begin{aligned}
& f_{p}(t, \boldsymbol{x}, \boldsymbol{G}, v)=f_{p}^{a p p}(t, \boldsymbol{x}, \boldsymbol{G}, v)=f_{p}^{d i v}(t, \boldsymbol{x}, \boldsymbol{G}, v) \\
& f_{p}^{a p p}(t, \boldsymbol{x}, \boldsymbol{G}, v)=\int f_{p}^{a p p}(t, \boldsymbol{x}, \boldsymbol{G}, v) \sin \theta \mathrm{d} \theta \mathrm{d} \varphi \\
& f_{p}^{d i v}(t, \boldsymbol{x}, \boldsymbol{G}, v)=\int f_{p}^{d i v}(t, \boldsymbol{x}, \boldsymbol{G}, v) \sin \theta \mathrm{d} \theta \mathrm{d} \varphi
\end{aligned}
$$

and $\nu, \theta$, and $\varphi$ are the spherical coordinates of the $\boldsymbol{v}$ vector. According to Equation (17), the

$f_{p}(t, \boldsymbol{x}, \boldsymbol{G}, v)$ function remains unchanged at time along the trajectory of the center of mass of the pair.

The lower moments of the pair distribution functions (18) are the local density of the number of pairs $n^{G}(t, \boldsymbol{x})$, the mean velocity of the centers of mass of pairs $\boldsymbol{U}^{G}(t, \boldsymbol{x})$, the $P_{i j}^{G}(t, \boldsymbol{x})$ tensor of stresses created as a result of movement of the centers of mass of pairs of particles, the $P_{i j}^{v}(t, \boldsymbol{x})$ tensor of stresses created because of relative movement of pair particles, the $\boldsymbol{q}^{G}(t, \boldsymbol{x})$ heat flux vector corresponding to the transfer of thermal energy because of movement of the centers of mass of pairs of particles, $\boldsymbol{q}^{v}(t, \boldsymbol{x})$ and $\boldsymbol{q}^{G v}(t, \boldsymbol{x})$ the and heat flux vectors corresponding to thermal energy transfer because of relative movement of particles in pairs. Along with $P_{i j}^{G}(t, \boldsymbol{x})$ and $P_{i j}^{v}(t, \boldsymbol{x})$ let us determine small stress tensors $p_{i j}^{G}(t, \boldsymbol{x})$ and $p_{i j}^{v}(t, \boldsymbol{x})$, and $p^{G}(t, \boldsymbol{x})$ and $p^{v}(t, \boldsymbol{x})$ pressure, and $T^{G}(t, \boldsymbol{x})$ and $T^{v}(t, \boldsymbol{x})$ temperature, $p^{G}=n k T^{G}, p^{v}=n k T^{v}$. The lower moments of the pair distribution functions (18) are determined by Equations (21)-(26) in [3]. The set of hydrodynamic values, namely, $n^{G}(t, \boldsymbol{x}), \boldsymbol{U}^{G}(t, \boldsymbol{x})$, $p^{G}(t, \boldsymbol{x}), p^{v}(t, \boldsymbol{x}), p_{i j}^{G}(t, \boldsymbol{x}), \boldsymbol{q}^{G}(t, \boldsymbol{x})$ and $\boldsymbol{q}^{v}(t, \boldsymbol{x})$ is constructed on the property of $\varphi_{2}^{(n)}(\boldsymbol{G}, v)$ $n=0,1, \cdots$ It is a set of the principal hydrodynamic values. The lower moments $p_{i j}^{v}(t, \boldsymbol{x})$ and $\boldsymbol{q}^{G v}(t, \boldsymbol{x})$ are not the principal hydrodynamic values.

The relations between the $f_{1}\left(t, \boldsymbol{x}_{1}, \boldsymbol{\xi}_{1}\right)$ one-particle distribution function and the pair distribution functions (18) from [3] can be used to find a relation between the one-particle distribution function and the pair distribution functions moments,

$$
\begin{aligned}
& n=n^{G} \quad \boldsymbol{U}=\boldsymbol{U}^{G} \\
& p=\frac{1}{2} p^{G}+\frac{1}{2} p^{v} \quad T=\frac{1}{2} T^{G}+\frac{1}{2} T^{v} \\
& P_{i j}=\frac{1}{2} P_{i j}^{G}+\frac{1}{2} P_{i j}^{v} \quad p_{i j}=\frac{1}{2} p_{i j}^{G}+\frac{1}{2} p_{i j}^{v} \\
& \boldsymbol{q}=\frac{1}{2} \boldsymbol{q}^{G}+\frac{5}{6} \boldsymbol{q}^{v}+\boldsymbol{q}^{G v}
\end{aligned}
$$

Here, $n=n(t, \boldsymbol{x})$ denotes the number densities of particles, $\boldsymbol{U}=\boldsymbol{U}(t, \boldsymbol{x})$ is the hydrodynamic velocity, $p=p(t, \boldsymbol{x})$ is the pressure, $T=T(t, \boldsymbol{x})$ is the tem- 
perature, $\quad p=n k T, \quad P_{i j}=P_{i j}(t, \boldsymbol{x})$ and $p_{i j}=p_{i j}(t, \boldsymbol{x})$ are the viscous stress tensors, and $\boldsymbol{q}=\boldsymbol{q}(t, \boldsymbol{x})$ is the heat flux.

A derivation of equations determining lower moments of the pair distribution functions is given in [3]. In [32], these hydrodynamics equations are written in the form,

$$
\begin{aligned}
& \frac{\partial}{\partial t}[m n]+\frac{\partial}{\partial x_{i}}\left[m n U_{i}\right]=0 \\
& \frac{\partial}{\partial t}\left[m n U_{i}\right]+\frac{\partial}{\partial x_{j}}\left[\frac{1}{2} p^{G} \delta_{i j}+\frac{1}{2} p_{i j}^{G}+m n U_{i} U_{j}\right]=0 \\
& \frac{\partial}{\partial x_{j}}\left[\frac{1}{2} p^{v} \delta_{i j}+\frac{1}{2} p_{i j}^{v}\right]=0 \\
& \frac{\partial}{\partial t}\left[\frac{3}{4} n k T^{G}+\frac{1}{2} m n \boldsymbol{U}^{2}\right] \\
& +\frac{\partial}{\partial x_{i}}\left[\frac{1}{2} q_{i}^{G}+\frac{5}{4} U_{i} n k T^{G}+\frac{1}{2} U_{j} p_{i j}^{G}+\frac{1}{2} m n U_{i} \boldsymbol{U}^{2}\right] \\
& =0 \\
& \frac{\partial}{\partial t}\left[\frac{3}{4} n k T^{v}\right]+\frac{\partial}{\partial x_{i}}\left[\frac{1}{2} q_{i}^{v}+\frac{3}{4} U_{i} n k T^{v}\right]=0 \\
& \frac{\partial}{\partial x_{i}}\left[q_{i}^{G v}+\frac{1}{3} q_{i}^{v}+\frac{1}{2} U_{i} p^{v}+\frac{1}{2} U_{j} p_{i j}^{v}\right]=0
\end{aligned}
$$

Taking (19) into account, we find that the sum of Equations (20b) and (20c) is none other than the equation of the conservation of momentum, and the sum of Equations (20d), (20e) and (20f) is none other than the equation of conservation of energy. Equation (20a) is the continuity equation. So, the equation for conservation of momentum separates into two equations, and the equation for conservation of energy, into three separate equations. In Equation (20), nonprincipal hydrodynamic values $p_{i j}^{v}$ and $q_{i}^{G v}$ are given by the equations,

$$
\begin{aligned}
p_{i j}^{v} & =-\tilde{\eta}\left(\frac{\partial U_{i}}{\partial x_{j}}+\frac{\partial U_{j}}{\partial x_{i}}-\frac{2}{3} \delta_{i j} \frac{\partial U_{k}}{\partial x_{k}}\right) \\
& -\frac{2 \tilde{\eta}}{3 p^{v}}\left(\frac{\partial q_{i}^{v}}{\partial x_{j}}+\frac{\partial q_{j}^{v}}{\partial x_{i}}-\frac{2}{3} \delta_{i j} \frac{\partial q_{k}^{v}}{\partial x_{k}}\right) \\
q_{i}^{G v} & =-\tilde{\lambda} \frac{\partial T}{\partial x_{i}}-\frac{\tilde{\lambda}}{5 p^{v}} \frac{\partial\left(p_{i j}^{G} T^{v}\right)}{\partial x_{j}} \\
& -\frac{4 \tilde{\lambda}}{15 p^{v}} \frac{m}{k}\left(q_{i}^{v} \delta_{j k}+q_{j}^{v} \delta_{i k}+q_{k}^{v} \delta_{i j}\right) \frac{\partial U_{k}}{\partial x_{j}}
\end{aligned}
$$

Here, $\tilde{\eta}$ is the dynamic viscosity coefficient and $\tilde{\lambda}=15 k \tilde{\eta} / 4 m$ is the heat conductivity coefficient.

Relations (19) and Equations (20), (21) contain seven lower principal hydrodynamic values, namely, $n^{G}(t, \boldsymbol{x})$,
$\boldsymbol{U}^{G}(t, \boldsymbol{x}), \quad p^{G}(t, \boldsymbol{x}), \quad p^{v}(t, \boldsymbol{x}), \quad p_{i j}^{G}(t, \boldsymbol{x}), \quad \boldsymbol{q}^{G}(t, \boldsymbol{x})$ and $\boldsymbol{q}^{v}(t, \boldsymbol{x})$. Just these values dominate in equations for measurable one-particle function moments (19). All the other higher principal hydrodynamic values (9) were omitted in Equations (19)-(21). An increase in the number of principal hydrodynamic values taken into account will cause the additional appearance of derivatives of all the higher principal moments (9) taken into account in both definitions (19) and Equations (20), (21). The lower one particle distribution function moments $n, \boldsymbol{U}$, and $p$ are written through the principal hydrodynamic values $n^{G}, \boldsymbol{U}^{G}, p^{G}(t, \boldsymbol{x})$ and $p^{v}(t, \boldsymbol{x})$. The higher measurable moments $p_{i j}$ and $\boldsymbol{q}$ are represented by linear combinations of principal and non-principal hydrodynamic values. The $p_{i j}^{G}$ stress tensor component is a principal hydrodynamic value. The first term of the $p_{i j}^{v}$ component is proportional to the tensor of deformation velocities $U_{i j}$ from Equation (21a), the remaining term of the component appears because of an increase in the number of principal hydrodynamic values. The first two components of the $\boldsymbol{q}$ heat flux vector, $\boldsymbol{q}^{G}$ and $\boldsymbol{q}^{v}$, are principal hydrodynamic values. The first term of the third component, $\boldsymbol{q}^{G v}$, is proportional to the temperature gradient from Equation (21b), and the other terms of the third component appear because of an increase in the number of principal hydrodynamic values.

The upper indexes "div" and "app" were omitted in the $p_{i j}^{v}$ and $q_{i}^{G v}$ hydrodynamic values because the right hand sides of Equations (19) and (21) do not experience any changes in calculations of moments with pair functions corresponding either to particles that approach each other, $f_{p}^{a p p}(t, \boldsymbol{x}, \boldsymbol{G}, \boldsymbol{v})$, or to particles that fly away from each other, $f_{p}^{d i v}(t, \boldsymbol{x}, \boldsymbol{G}, \boldsymbol{v})$ [3].

The analysis performed in [35] showed that hydrodynamic Equations (20), (21) could be rigorously reduced to classic hydrodynamics equations only when the state of the system weakly deviated from thermodynamic equilibrium. In particular, in the $\mathrm{Re} \ll 1$ limit, the $p_{i j}^{G}(t, \boldsymbol{x})$ main hydrodynamic value and the $p_{i j}^{v}(t, \boldsymbol{x})$ value are equal. Moreover, the second term on the righthand side of Equation (21a), which is responsible for viscous stresses caused by heat fluxes, becomes negligibly small compared with the first term. It follows that, as is shown in [35], the Newton law [29] is only valid in the $\mathrm{Re} \ll 1$ limit.

An indefinite $\lambda(t, x)$ parameter is the characteristic free path. An indefinite $\lambda(t, x)$ parameter is introduced at the stage of the formation of pair distribution functions (15). The characteristic time between collisions $\tau(t, \boldsymbol{x})=\lambda(t, \boldsymbol{x}) / v$ is contained in resulting kinetic equations and hydrodynamics equations [3]. The limit of weak non-equilibrium allows us to find the proportionality factor between the $\tilde{\eta}$ dynamic viscosity coefficient and $\tau$, 


$$
\tau=2 \tilde{\eta} / p^{v}
$$

The $\tilde{\eta}$ coefficient can be calculated with arbitrary accuracy by solving the Boltzmann equation with the use of the Chapman-Enscog method for an arbitrary law of interparticle interactions [31].

In calculations of the right hand sides of the equations for the pair functions (18), collision integrals were calculated in [3] for a gas consisting of rigid spheres with diameter $d$. This case has exceptional advantages, because, in integration in velocities $\boldsymbol{G}$ and $\boldsymbol{v}$ with the $\varphi_{2}^{(n)}(\boldsymbol{G}, v) \quad n=0,1, \cdots$, weight function, the collision integrals of kinetic equations are expressed exactly in terms of the moments of pair distribution functions. The classic Boltzmann collision integral offers similar advantages to the case of so-called Maxwell molecules [36]. The structure of the derived hydrodynamics Equations (20) and (21) does not depend on the form of the law of structureless particle interactions. The interaction law only influences the values of transfer coefficients. It follows that system (20) and (21) is valid for the description of gas flows with an arbitrary law of interaction of structureless particles.

In the derivation of hydrodynamics Equations (19)(21), only terms linear in $\tau$ are retained. At the hydrodynamic description stage, the omitted terms correspond to so-called Barnett accuracy $[31,36]$. In follows that hydrodynamics Equations (20) and (21) presented above correspond to Navier-Stokes accuracy of description.

The system of equations determining lower moments of the pair distribution functions $(20,21)$ is not closed. Let us multiply the Equation (17) for the $f_{p}(t, \boldsymbol{x}, \boldsymbol{G}, v)$ pair function by lower invariants of binary particle collision, $\varphi_{2}^{(n)}(\boldsymbol{G}, v)$, and integrate the result with respect to velocities $\mathbf{G}$ and $v$. By virtue of definition of lower moments of pair functions [3], we then have hydrodynamic Equations (20a, b, d, e). Thus, Equation (17) is equivalent to several equations, namely, Equations (20a, $\mathrm{b}, \mathrm{d}, \mathrm{e})$. According to (17), the $f_{p}(t, \boldsymbol{x}, \boldsymbol{G}, v)$ function remains unchanged at time along the trajectory of the center of mass of the pair. This property creates the additional relations between the principal hydrodynamic values. The $\boldsymbol{U}^{G}, \boldsymbol{q}^{G}$ and $\boldsymbol{q}^{v}$ vectors, and the $p_{i j}^{G}$ tensor are multicomponent principal hydrodynamic values. It turned out that the use of the conservation property (17) is accompanied by the appearance of additional relations between each vector and tensor components. Generally, just these relations must close the system $(20,21)$ of hydrodynamics equations.

However, a problem on flow around a fixed solid sphere was solved in [4,5] by another manner. In [4], the solution to Equation (17) was built. The first integrals of Equation (17) for a stationary system are the $F_{x y}(\boldsymbol{G}, \boldsymbol{x})$, $F_{y z}(\boldsymbol{G}, \boldsymbol{x})$, and $F_{z x}(\boldsymbol{G}, \boldsymbol{x})$ functions,

$$
\begin{aligned}
& F_{x y}=G_{x} y-G_{y} x \quad F_{y z}=G_{y} z-G_{z} y \\
& F_{z x}=G_{x} z-G_{z} x \\
& \boldsymbol{G}=\left(G_{x}, G_{y}, G_{z}\right), \quad \boldsymbol{x}=(x, y, z)
\end{aligned}
$$

According to [4], a solution to Equation (17) in the problem with time-independent boundary conditions should be sought in the form of a series of the products of the first integrals,

$$
\begin{aligned}
& f_{p}(t, \boldsymbol{x}, \boldsymbol{G}, v)=\left(\frac{m}{2 \pi k T_{0}}\right)^{3} \exp \left(-\frac{m \boldsymbol{G}^{2}}{k T_{0}}\right) \\
& \cdot \exp \left(-\frac{m v^{2}}{4 k T_{0}}\right) \sum_{i=1}^{S} c_{i} \varphi_{2}^{(j)} F_{x y}^{l} F_{y z}^{r} F_{z x}^{s} \\
& i=(j, l, r, s)
\end{aligned}
$$

Here, $T_{0}$ is the temperature of the unperturbed medium and the $c_{i}$ coefficients generally depend on $t$. Substituting the distributions of the principal hydrodynamic values calculated by Equation (24) into the hydrodynamic Equations (20c, f) yields a nonlinear system of differential equations for the $C_{i}$ coefficients,

$$
\frac{\partial C_{i}}{\partial t}=F_{i}\left(C_{1}, \cdots, C_{S}\right) \quad i=1, \cdots, S
$$

Relations between the $C_{i}$ dimensionless coefficients and the $c_{i}$ dimensional coefficients are given in [4].

\section{Conclusions}

Figure 1 represents observed flow pictures around a sphere. Experiment records three stable flows: $\boldsymbol{U}_{s 0}^{\exp }(\boldsymbol{x})$, $\boldsymbol{U}_{s 1}^{\exp }(\boldsymbol{x})$, and $\boldsymbol{U}_{s 2}^{e x p}(t, \boldsymbol{x})$. Each of these three flows after losing the stability starts to evolve in its own direction qualitatively different from the others. These directions are schematically shown by three horizontal branches. At these branches experiment records at least six vortex shedding modes: $\boldsymbol{W}_{s 0}^{\exp }(t, \boldsymbol{x})$ and $\boldsymbol{Q}_{s 0}^{\exp }(t, \boldsymbol{x})$; $\boldsymbol{V}_{s 1}^{\text {exp }}(t, \boldsymbol{x}) ; \boldsymbol{V}_{s 2}^{\text {exp }}(t, \boldsymbol{x}), \boldsymbol{W}_{s 2}^{\text {exp }}(t, \boldsymbol{x})$ and $\boldsymbol{Q}_{s 2}^{\exp }(t, \boldsymbol{x})$.

The integration of the Navier-Stokes equations for the problem of flow past a sphere $[15,16,18-21]$ gives two stable solutions, $\boldsymbol{U}_{s 0}^{\text {cal }}(\boldsymbol{x})$ and $\boldsymbol{U}_{s 1}^{\text {cal }}(\boldsymbol{x})$, and the $\boldsymbol{U}_{s 2}^{c a l}(t, \boldsymbol{x})$ stable limiting cycle. These solutions satisfactorily reproduce three stable flows observed experimentally, $\boldsymbol{U}_{s 0}^{\exp }(\boldsymbol{x}), \boldsymbol{U}_{s 1}^{\exp }(\boldsymbol{x})$, and $\boldsymbol{U}_{s 2}^{\exp }(t, \boldsymbol{x})$. Calculations were, however, incapable of reproducing any of the six vortex shedding modes. The calculation leads the instability development process in the direction given by dashed slanting line (Figure 1). The dashed slanting line separates stable solutions from unstable ones, that is, the dashed line gives the boundary of the instability field. So, solutions to the classic hydrodynamics equations successfully reach the boundary of the instability field. As Reynolds grows, these solutions move along the bound- 
ary of the field. However, the solutions to the classic hydrodynamics equations are unable to cross this boundary and to pass to the instability field. There are no numerical experiments on flow around different bluff bodies in which this boundary is crossed. Most likely, the reason for simulation failure is the Navier-Stokes equations themselves.

Analysis of the multimoment hydrodynamics equations [35] substantiates the suggestion that the applicability scope of classic hydrodynamics is limited, in particular, by small Reynolds number values, $\mathrm{Re} \ll 1$. Nevertheless, the calculated stable solutions to the NavierStokes equations, $\boldsymbol{U}_{s 0}^{\text {cal }}(\boldsymbol{x}), \boldsymbol{U}_{s 1}^{\text {cal }}(\boldsymbol{x})$ and $\boldsymbol{U}_{s 2}^{\text {cal }}(t, \boldsymbol{x})$, satisfactorily reproduce stable flows around a sphere $\boldsymbol{U}_{s 0}^{\exp }(\boldsymbol{x}), \boldsymbol{U}_{s 1}^{\exp }(\boldsymbol{x})$, and $\boldsymbol{U}_{s 2}^{\exp }(t, \boldsymbol{x})$ recorded experimentally at moderately high Reynolds number values, reaching a few hundreds. This means that the error in stable solutions to the Navier-Stokes equations is not large and cannot distort calculated flow pictures noticeably. This error, however, grows very rapidly after the loss of stability. This is explained by the tendency of nonlinear equations toward causing the divergence of close solutions even in a limited phase space region. This sensitivity to initial conditions was called the Lorentz butterfly effect. The loss of instability development direction is the result of error growth, i.e., the classic hydrodynamics equations become helpless in producing of regular nonstationary periodic flows appearing after the attainment of a certain critical Reynolds number value.

The numerical integration of the multimoment hydrodynamics equations in the problem on a flow past a sphere $[4,5]$ shows that the solutions to these equations cross the boundary and enter the instability field. Let $\bar{C}_{i}^{(\alpha)}$ be a stationary $\alpha$-solution of the system (25) and $\delta C_{i}^{(\alpha)}(t)$ be a perturbation of the $\bar{C}_{i}^{(\alpha)}$ solution, $C_{i}(t)=\bar{C}_{i}^{(\alpha)}+\delta C_{i}^{(\alpha)}(t)$. The retention of three-lower axisymmetric terms in expansion (24) leads to the classic Stokes solution valid in the $\mathrm{Re} \ll 1$ limit [29]. For advancement up the Re scale, twenty lower axisymmetric expansion (24) terms are retained in [4]. The $\bar{C}_{i}^{(0)}, i=1, \cdots, 20$, stationary solution to the nonlinear algebraic system of twenty equations gives the stationary distribution of the hydrodynamic values (19). According to the $\bar{C}_{i}^{(0)}, i=1, \cdots, 20$, solution, an axisymmetric recirculating zone is formed in the wake behind a sphere at $\operatorname{Re} \sim 20$. This recirculating zone has the shape of an axisymmetric toroidal ring. It expands as Re grows but its shape remains unchanged. The calculated distribution $\boldsymbol{U}_{s 0}^{\text {cal }}(\boldsymbol{x})$ satisfactorily reproduces the $\boldsymbol{U}_{s 0}^{\text {exp }}(\boldsymbol{x})$ axisymmetric flow.

The $\bar{C}_{i}^{(0)}, i=1, \cdots, 20$, solution remains stable up to a certain critical $\mathrm{Re}_{0}^{*}$ value. This means that the $\delta C_{i}^{(0)}(t)$ small perturbations of the solution
$\bar{C}_{i}^{(0)}, i=1, \cdots, 20$, are damped at $\operatorname{Re}<\operatorname{Re}^{*}$. The passage of $\mathrm{Re}_{0}^{*}$ is accompanied by the $\bar{C}_{i}^{(0)}, i=1, \cdots, 20$, solution stability loss. Starting with $t=0$, the

$\delta C_{i}^{(0)}(t), i=1, \cdots, 20$ small axisymmetric perturbations begin to increase exponentially at $\operatorname{Re}>\mathrm{Re}_{0}^{*}$. The $\delta C_{i}^{(0)}(t)$ perturbations grow up to time $t_{*}>0$. At $t=t_{*}$, the $\delta C_{i}^{(0)}(t)$ solution is cut off. The reason for this is analyzed in [4].

It turned out that another solution to the nonlinear system of differential Equations (25) existed in the neighborhood of the cut-off point. It is the

$\delta C_{i}^{+(0)}(t), i=1, \cdots, 20$, solution of the so-called system of the reversed hydrodynamics equations [35]. According to [37], the law of large numbers is violated near singular points (bifurcations, regions of the coexistence of several stable solutions, etc.), and large fluctuations may appear in the system. In conformity with [37], a large fluctuation causes the transfer of the system from the cut-off point to the $\delta C_{i}^{+(0)}(t), i=1, \cdots, 20$, solution at time $t=t_{*}$. At time $t=2 t_{*}$, the $\delta C_{i}^{+(0)}(t)$ solution reaches the neighborhood of the $\bar{C}_{i}^{(0)}, i=1, \cdots, 20$, stationary solution. At time $t=2 t_{*}$ further evolution of the $\delta C_{i}^{+(0)}(t), i=1, \cdots, 20$, solution is finished. The $\delta C_{i}^{(0)}(t), i=1, \cdots, 20$, solution exists at $0 \leq t \leq t_{*}$, the $\delta C_{i}^{+(0)}(t), i=1, \cdots, 20$, solution exists at $t_{*} \leq t \leq 2 t_{*}$. A fluctuation causes the transfer of the system from the $\bar{C}_{i}^{(0)}+\delta C_{i}^{+(0)}(t), i=1, \cdots, 20$, solution to the $\bar{C}_{i}^{(0)}+\delta C_{i}^{(0)}(t), i=1, \cdots, 20$, solution at time $t=2 t_{*}$. The $\bar{C}_{i}^{(0)}, i=1, \cdots, 20$ solution is unstable. It follows that small axisymmetric $\delta C_{i}^{(0)}(t), i=1, \cdots, 20$, perturbations begin to grow starting with time $t=2 t_{*}$. This process is repeated periodically.

The evolution of the $\bar{C}_{i}^{(0)}+\delta C_{i}^{(0)}(t)$ and $\bar{C}_{i}^{(0)}+\delta C_{i}^{+(0)}(t), i=1, \cdots, 20$, solution satisfactorily reproduces the $\boldsymbol{V}_{s 0}^{\exp }(t, \boldsymbol{x})$ periodic axisymmetric pulsations from [6,10]. According to the $\bar{C}_{i}^{(0)}+\delta C_{i}^{(0)}(t)$ and $\bar{C}_{i}^{(0)}+\delta C_{i}^{+(0)}(t), i=1, \cdots, 20$, solution, toroidal recirculating zone in the near wake behind a sphere begins to pulsate periodically after the attainment of the Reynolds number $\mathrm{Re}_{0}^{*}$. The recirculating zone remains toroidal during pulsations, and its forefront is firmly fixed on the sphere. The pulsating flow remains axisymmetric. Because of the absence of the detachment of the recirculating zone periphery, there is no vortex loop street in the far wake behind a sphere.

The scenario of instability appearance, $R e>\mathrm{Re}_{0}^{*}$, $[4,5]$, has no analogues. Currently, we have no alternative to this scenario, because Landau's scenario based on solutions to the Navier-Stokes equations [15,16,18-21] fails to describe experiment.

The crossing of the instability field boundary is accompanied by the appearance of uncommon acts in the scenario of instability development. It turned out that the 
exponential growth of small perturbations of stationary unstable solution is not infinite. At some point the nonstationary solution is cut off and the growth of perturbations is completed. After the break the system finds extremely unlike direction of evolution. It starts a returning motion in the direction of the unstable stationary solution. As a result, the system after losing the stability does not find a new stable position, i.e., after crossing the boundary of the instability field, the evolution of the system takes place only within the instability field. Such a scenario differs from the ideas of classic hydrodynamics, which interprets the development of instability in terms of bifurcations from one stable state to another stable state.

The importance of the entropy in interpretation of instability is revealed in [5]. It turns out that the explanation of instability appearance lies in change in entropy balance. Namely, an open system with time-independent boundary conditions has a steady state while entropy production in it exceeds entropy outflow through the surface confining the system. Then, any fluctuation generated by the system fades out. As soon as the entropy is removed through the confining surface faster than it is produced in the system, any fluctuation generated by the system begins to grow. The system becomes unstable.

Further studies [38] show that reproduction of the $\boldsymbol{W}_{s 0}^{e x p}(t, \boldsymbol{x})$ and $\boldsymbol{Q}_{s 0}^{e x p}(t, \boldsymbol{x})$ vortex shedding regimes, $\operatorname{Re}>\operatorname{Re}_{0}^{* *}$, leads to new scenario acts that do not have analogues. Moreover, the $S_{p}(t)$ pair entropy of system begins to play the key role to predict the instability development direction. The calculation of the system evolution becomes impossible without participation of the entropy. The multimoment hydrodynamics equations find a large number of ways for system evolution. And the $S_{p}(t)$ pair entropy shows the single way, in which the system evolution moves.

So, the perspectives for removal the discrepancies between calculations and experiment for unstable regimes are revealed along the direction on increase of the principal hydrodynamic values number when deriving the hydrodynamics equations.

\section{REFERENCES}

[1] I. V. Lebed, "On the Inapplicability of Navier-Stokes Equations to Interpreting the Turbulence," Physica A, Vol. 315 , No. 1-2, 2002, pp. 228-235. doi:10.1016/S0378-4371(02)01254-2

[2] I. V. Lebed and S. Y. Umanskii, "The Appearance and Development of Turbulence in a Flow Past a Sphere: Problems and the Existing Approaches to Their Solution," RJ Physical Chemistry B, Vol. 1, No. 1, 2007, pp. 52-73.

[3] I. V. Lebed, "Method of Two-Particle Distribution Functions. Hydrodynamic Equations," Chemical Physics Reports, Vol. 15, No. 6, 1996, pp. 861-883.
[4] I. V. Lebed, "The Method of Pair Functions as Applied to the Problem of a Flow around a Quiescent Solid Sphere," Chemical Physics Reports, Vol. 16, No. 7, 1997, pp. 1263-1301.

[5] I. V. Lebed, "About the Behavior of the Entropy of a Gas Flow Losing Its Stability," Chemical Physics Reports, Vol. 17, No. 3, 1998, pp. 411-439.

[6] S. Taneda, "Experimental Investigation of the Wake behind a Sphere at Low Reynolds Numbers," Journal of the Physical Society of Japan, Vol. 11, 1956, pp. 1104-1108. doi:10.1143/JPSJ.11.1104

[7] H. Sakamoto and H. Haniu, "The Formation Mechanism and Shedding Frequency of Vortices from a Sphere in Uniform Shear Flow," Journal of Fluid Mechanics, Vol. 287, 1995, pp. 151-171. doi:10.1017/S0022112095000905

[8] R. H. Magarvey and R. L. Bishop, "Transition Ranges for Three-Dimensional Wakes," Canadian Journal of Physics, Vol. 39, No. 10, 1961, pp. 1418-1422. doi:10.1139/p61-169

[9] E. Achenbach, "Vortex Shedding from Spheres," Journal of Fluid Mechanics, Vol. 62, No. 2, 1974, pp. 209-221. doi: $10.1017 / \mathrm{S} 0022112074000644$

[10] J. M. Chomaz, P. Bonneton and E. J. Hopfinger, "The Structure of the Near Wake of a Sphere Moving Horizontally in a Stratified Fluid," Journal of Fluid Mechanics, Vol. 254, 1993, pp. 1-21. doi:10.1017/S0022112093002009

[11] J. Gerrard, "The Wakes of Cylindrical Bluff Bodies at Low Reynolds Numbers," Philosophical Transactions of the Royal Society of London A, Vol. 288, No. 1354, 1978 , pp. 351-382. doi:10.1098/rsta.1978.0020

[12] M. M. Zdravkovich, "Smoke Observation of the Formation of a Karman Vortex Street," Journal of Fluid Mechanics, Vol. 37, No. 3, 1969, pp. 491-496. doi: $10.1017 / \mathrm{S} 0022112069000681$

[13] B. E. Eaton, "Analysis of Laminar Vortex Shedding behind a Circular Cylinder by Computer-aided Flow Visualization," Journal of Fluid Mechanics, Vol. 180, 1987, pp. 117-145. doi:10.1017/S0022112087001757

[14] J. Cohen, I. G. Shukhman, M. Karp and J. Philip, “An Analytical-Based Method for Studying the Nonlinear Evolution of Localized Vortices in Planar Homogenous Shear Flows," Journal of Computational Physics, Vol. 229, No. 20, 2010, pp. 7765-7773. doi:10.1016/j.jcp.2010.06.035

[15] I. Kim and A. J. Pearlstein, "Stability of the Flow Past a Sphere," Journal of Fluid Mechanics, Vol. 211, 1990, pp. 73-83. doi:10.1017/S0022112090001501

[16] R. Natarajan and A. Acrivos, "The Instability of the Steady Flow Past Spheres and Disks," Journal of Fluid Mechanics, Vol. 254, 1993, pp. 323-344. doi: $10.1017 / \mathrm{S} 0022112093002150$

[17] H. G. Schuster, "Deterministic Chaos," Physik-Verlag, Weinheim, 1984.

[18] A. G. Tomboulides, S. A. Orszag and G. E. Karniadakis, "Direct and Large-Eddy Simulations of Axisymmetric Wakes," AIAA Paper, No. 93-9546, 1993. 
[19] R. Mittal, "Planar Symmetry in the Unsteady Wake of a Sphere," AIAA Journal, Vol. 37, No. 3, 1999, pp. 388-400. $\underline{\text { doi: } 10.2514 / 2.722}$

[20] T. A. Johnson and V. S. Patel, "Flow Past a Sphere Up to a Reynolds Number of 300," Journal of Fluid Mechanics, Vol. 378, 1999, pp. 19-70. doi: $10.1017 / \mathrm{S} 0022112098003206$

[21] A. G. Tomboulides and S. A. Orszag, "Numerical Investigation of Transitional and Weak Turbulent Flow Past a Sphere," Journal of Fluid Mechanics, Vol. 416, 2000, pp. 45-73. doi:10.1017/S0022112000008880

[22] C. P. Jackson, "A Finite-Element Study of the Onset of Vortex Shedding in Flow Past Variously Shaped Bodies," Journal of Fluid Mechanics, Vol. 182, 1987, pp. 23-45. doi:10.1017/S0022112087002234

[23] K. Hannemann and H. Oertel Jr., "Numerical Simulation of the Absolutely and Convectively Unstable Wake," Journal of Fluid Mechanics, Vol. 199, 1989, pp. 55-88. doi:10.1017/S0022112089000297

[24] P. J. Strykowski and K. Hannemann, "Temporal Simulation of the Wake behind a Circular Cylinder in the Neighborhood of the Critical Reynolds Number," Acta Mechanica, Vol. 90, No. 1-4, 1991, pp. 1-20. doi:10.1007/BF01177395

[25] G. K. Batchelor, "An Introduction to Fluid Dynamics," Cambridge at the University Press, Cambridge, 1970.

[26] V. Gushchin and P. Matyushin, "Vortex Formation Mechanisms in the Wake behind a Sphere for $200<\mathrm{R} e<380$," Fluid Dynamics, Vol. 41, 2006, pp. 795-809. doi:10.1007/s10697-006-0096-X

[27] S. Baghery, P. Schlatter, P. Schmid and D. Henningson, "Global Stability of a Jet Cross-Flow," Journal of Fluid Mechanics, Vol. 624, 2009, pp. 33-44. doi:10.1017/S0022112009006053

[28] J. Jeong and F. Hussain, "On the Identification of a Vor- tex," Journal of Fluid Mechanics, Vol. 285, 1995, pp. 6994. doi:10.1017/S0022112095000462

[29] L. G. Loitsyanskii, "Mechanics of Liquids and Gases," Pergamon, Oxford, 1966.

[30] R. L. Liboff, "Introduction to the Theory of Kinetic Equations," Wiley, New York, 1969.

[31] J. H. Ferziger and H. G. Kaper, "Mathematical Theory of Transport Processes in Gases," North-Holland Publishing Company, Amsterdam, 1972.

[32] I. V. Lebed and S. Y. Umanskii, "On the Possibility of Improving Classic Hydrodynamics Equations by an Increase in the Number of Hydrodynamic Values," $R J$ Physical Chemistry B, Vol. 6, No. 1, 2012, pp. 149-162.

[33] I. V. Lebed, "Derivation of the Equations for Pair Distribution Functions," Chemical Physics Reports, Vol. 14, No. 5, 1995, pp. 599-615.

[34] I. V. Lebed, "Equations of Pair Distribution Functions," Chemical Physics Letters, Vol. 165, No. 2-3, 1990, pp. 226- 228. doi:10.1016/0009-2614(90)85433-D

[35] I. V. Lebed, "Hydrodynamic Equations Stemming from Two Particle Distributions in the Limit of Weak Nonequilibrium. Analysis of Invertibility of Equations," Chemical Physics Reports, Vol. 15, No. 12, 1996, pp. 17251750 .

[36] H. Grad, "About Kinetic Theory of Rarefied Gases," Communications on Pure and Applied Mathematics, Vol. 2, No. 4, 1949, pp. 331-407. doi:10.1002/cpa.3160020403

[37] I. Prigogine, "From Being to Becoming," Freeman, San Francisco, 1980.

[38] I. V. Lebed, “The Development of Instability in Problem on Flow around a Sphere," RJ Physical Chemistry B, Vol 8, No. 1, 2014, in Press. 